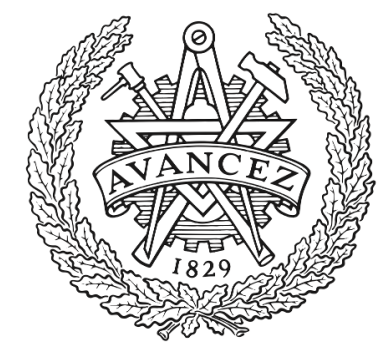

CHALMERS

UNIVERSITY OF TECHNOLOGY

\title{
Signatures of van der Waals binding: A coupling-constant scaling analysis
}

Downloaded from: https://research.chalmers.se, 2023-04-26 15:13 UTC

Citation for the original published paper (version of record):

Jiao, Y., Schröder, E., Hyldgaard, P. (2018). Signatures of van der Waals binding: A coupling-constant scaling analysis. Physical Review B, 97(8).

http://dx.doi.org/10.1103/PhysRevB.97.085115

N.B. When citing this work, cite the original published paper. 


\title{
Signatures of van der Waals binding: A coupling-constant scaling analysis
}

\author{
Yang Jiao, * Elsebeth Schröder, and Per Hyldgaard \\ Department of Microtechnology and Nanoscience - MC2, Chalmers University of Technology, SE-41296 Gothenburg, Sweden
}

(Received 2 October 2017; revised manuscript received 3 January 2018; published 12 February 2018)

\begin{abstract}
The van der Waals (vdW) density functional (vdW-DF) method [Rep. Prog. Phys. 78, 066501 (2015)] describes dispersion or vdW binding by tracking the effects of an electrodynamic coupling among pairs of electrons and their associated exchange-correlation holes. This is done in a nonlocal-correlation energy term $E_{\mathrm{c}}^{\mathrm{nl}}$, which permits density functional theory calculation in the Kohn-Sham scheme. However, to map the nature of vdW forces in a fully interacting materials system, it is necessary to also account for associated kinetic-correlation energy effects. Here, we present a coupling-constant scaling analysis, which permits us to compute the kinetic-correlation energy $T_{\mathrm{c}}^{\mathrm{nl}}$ that is specific to the vdW-DF account of nonlocal correlations. We thus provide a more complete spatially resolved analysis of the electrodynamical-coupling nature of nonlocal-correlation binding, including $\mathrm{vdW}$ attraction, in both covalently and noncovalently bonded systems. We find that kinetic-correlation energy effects play a significant role in the account of vdW or dispersion interactions among molecules. Furthermore, our mapping shows that the total nonlocal-correlation binding is concentrated to pockets in the sparse electron distribution located between the material fragments.
\end{abstract}

DOI: 10.1103/PhysRevB.97.085115

\section{INTRODUCTION}

Many-body effects are essential for an accurate description of materials bonds. Such correlation effects must be accurately reflected in density functional theory (DFT) as we seek approximate evaluations of ground-state expectation values, $\langle\hat{T}\rangle$ and $\langle\hat{V}\rangle$, of operators for the kinetic energy $\hat{T}$ and for the electronelectron interaction $\hat{V}$. This is clear, for example, because dispersion or van der Waals ( $\mathrm{vdW}$ ) interactions arise from an electrodynamical coupling among collective excitations [1-6]. In the Kohn-Sham (KS) scheme [7], for efficient, in principle, exact DFT calculations, we handle all many-body effects by a trick. We focus on an independent-particle approximation, the KS kinetic-energy term $T_{\mathrm{KS}}$, while embedding the difference, termed the kinetic-correlation energy,

$$
T_{\mathrm{c}}=\langle\hat{T}\rangle-T_{\mathrm{KS}}
$$

in the exchange-correlation $(\mathrm{XC})$ energy $E_{\mathrm{xc}}$. However, the kinetic-correlation energy, Eq. (1), can still be unmasked as a functional $T_{c}[n]$ of the electron density $n(\mathbf{r})$ using a formally exact scaling analysis [8-11]. An evaluation of $E_{\mathrm{xc}}[n]-T_{\mathrm{c}}[n]$ is equivalent to correcting $\langle\hat{V}\rangle$ beyond the Hartree approximation [8] and thus allows an exploration of many-electron interaction effects.

The van der Waals (vdW) density functional (vdW-DF) method for general-purpose DFT calculations relies on truly nonlocal formulations $E_{\mathrm{xc}}[n]$, Refs. [12-20]. The vdW-DF functional design can be seen as a systematic extension of the local density approximations (LDA) and of the generalized gradient approximation (GGA). In its original and most commonly used form $[15,18]$, it relies on the same manybody perturbation theory analysis $[5,16]$ that underpins the

\footnotetext{
*yang.jiao@chalmers.se
}

formulations of PBE [21] and PBEsol [22], and it adheres to the same fundamental principle, that physics constraints, including charge [15] and current $[6,15]$ conservation, should guide the XC functional design [14,23,24]. However, as part of a rationale for constraint-based GGA, Langreth and Vosko showed that a gradient-corrected formulation of correlation cannot naturally account for vdW interactions [5]. The vdWDF method overcomes that limitation, noting that electrons and their associated GGA-type XC holes themselves form dipole systems with internal dynamics $[3,4,6,12,25,26]$. The vdWDF method tracks screening effects produced by the mutual electrodynamical coupling of such virtual dipoles. It thus extends GGA within the vdW-DF framework, capturing screened dispersion binding $[3,4]$ by summing coupling-induced shifts in the collective plasmon excitations [1,6].

The vdW-DF method is computationally efficient since the dispersion-energy gains [6] are evaluated in a truly nonlocalcorrelation energy term $E_{\mathrm{c}}^{\mathrm{nl}}$ that is an explicit functional of the density [15,27]. This is done by using the adiabatic-connection formula $[2,28,29]$ (ACF) for the exact XC functional to define an effective dielectric function $\kappa[6,15,20]$ and by expanding $\kappa$ in terms of a plasmon-pole approximation that reflects the response corresponding to an internal semilocal functional $[6,15,17,23] E_{\mathrm{xc}}^{\mathrm{in}}$. In the original general-geometry vdW-DF [15] and in the recent consistent-exchange vdW-DF-cx [18] formulations, ${ }^{1}$ this internal function comprises LDA with gradient corrections defined by analysis of screened exchange $[5,15,16,19]$. The total functional specification $[15,18]$

$$
E_{\mathrm{xc}}^{\mathrm{vdW}-\mathrm{DF}}[n]=E_{\mathrm{xc}}^{\mathrm{in}}+E_{\mathrm{c}}^{\mathrm{nl}}+\delta E_{\mathrm{x}}^{0},
$$

\footnotetext{
${ }^{1}$ In the case of vdW-DF2 [17] by a formulation that reflects an exchange-scaling to the high-density limit.
} 
generally also contains a cross-over term $\delta E_{\mathrm{x}}^{0}$ that contains nothing but gradient-corrected exchange [20,24]. The total exchange functional $E_{\mathrm{x}}$ is semilocal; the correlation part of the functional, $E_{\mathrm{c}}$, comprises LDA correlation $E_{\mathrm{c}}^{\mathrm{LDA}}\left(\right.$ from $\left.E_{\mathrm{xc}}^{\mathrm{in}}\right)$ and $E_{\mathrm{c}}^{\mathrm{nl}}$.

An elegant illustration of the many-body physics nature of vdW binding can be obtained by computing the spatially resolved component [30,31]

$$
e_{\mathrm{c}}^{\mathrm{nl}}[n](\mathbf{r})=\frac{n(\mathbf{r})}{2} \int_{\mathbf{r}^{\prime}} \Phi\left(n(\mathbf{r}), \nabla n(\mathbf{r}), n\left(\mathbf{r}^{\prime}\right), \nabla n\left(\mathbf{r}^{\prime}\right)\right) n\left(\mathbf{r}^{\prime}\right),
$$

of the total vdW-DF nonlocal-correlation energy

$$
E_{\mathrm{c}}^{\mathrm{nl}}[n]=\int_{\mathbf{r}} e_{\mathrm{c}}^{\mathrm{nl}}[n](\mathbf{r}) .
$$

The spatial resolution (4) is a natural extension of how we normally analyze total-energy contributions arising from the semilocal components of the XC energy [2,11,15,21]. The spatially resolved energy (3) is given by the vdW-DF kernel $[15,32] \Phi$ for which there exist both formal analysis [16] and an efficient evaluation scheme [27]. With Eq. (3), one can track and understand binding-induced changes $\Delta e_{\mathrm{c}}^{\mathrm{nl}}(\mathbf{r})$, for example, for benzene adsorption on graphene [31]. The mapping confirms that the dominant contributions to the vdW binding arise in the regions of sparse [33] (but not vanishingly low) electron density between molecules and surfaces [6,14,34-36].

In this paper, we seek a characterization of electronelectron interaction effects that underpin vdW attraction between molecules. Many computational descriptions of the vdW attraction build on a discussion of the electron response and dielectric function in the physical, fully interacting system $[1,3,4,12-14,25,26,37-50]$, although the actual response behavior is sometimes approximated by an independent-particle description. The ACF specifies the exact XC functional as an average over the electron response, denoted $\chi_{\lambda}$, that reflects a ramping $(0<\lambda<1)$ of assumed electron-electron interaction strengths, $\hat{V}_{\lambda}=\lambda \hat{V} \quad[2,11,28,29,51]$. This ACF view is explicitly maintained in vdW density functionals $[15,19,20,24,52,53]$, which track the vdW binding produced by plasmon-energy shifts [1,3] in a $\mathrm{KS}$ framework [2,6]. However, the electrodynamical-coupling mechanism for vdW attraction $[3,4]$ is at work in the physical system, i.e., at full coupling-constant strength $\lambda=1$. For a more complete mapping of the nature of $\mathrm{vdW}$ attraction [3], we therefore seek to (a) compute an XC energy, denoted $E_{\mathrm{xc}, \lambda=1}[n]$, that instead reflects the physical response $\chi_{\lambda=1}$, and (b) extract and study the component, denoted $E_{\mathrm{c}, \lambda=1}^{\mathrm{nl}}[n]$, that corresponds to nonlocal-correlation effects in $\chi_{\lambda=1}$.

Our central observation is that such information is directly available from the vdW-DF functional form, Eq. (2), by applying the formally exact coupling-constant scaling analysis $[8,9,54]$ on the vdW-DF method. The formal analysis rests on density scaling, which provides a complete specification of the would-be XC energy $E_{\mathrm{xc}, \lambda}[n]$ that reflects the response function $\chi_{\lambda}$ assuming only that the $\lambda$-averaged response defines the specific $E_{\mathrm{xc}}[n]$ form; the analysis can be made for a given problem once we know the self-consistent solution density $n(\mathbf{r})$. We present details of how to extend the scaling analysis from semilocal functionals $[10,11,55,56]$ to the truly nonlocalcorrelation term $E_{\mathrm{c}}^{\mathrm{nl}}[n]$ of the vdW-DF method.
We note that the formal scaling analysis permits calculations of the kinetic-correlation energy [8], Eq. (1). For practical calculations, we present a code, termed PPACF, that computes the component

$$
T_{\mathrm{c}}^{\mathrm{nl}}[n]=\int_{\mathbf{r}} t_{\mathrm{c}}^{\mathrm{nl}}[n](\mathbf{r}),
$$

which is specific to $E_{\mathrm{c}}^{\mathrm{nl}}[n]$. Equation (5) is also combined with the known coupling-constant scaling analysis for LDA correlation [8-10,54], for a full specification of the kineticcorrelation energy

$$
T_{\mathrm{c}}[n]=\int_{\mathbf{r}} t_{\mathrm{c}}[n](\mathbf{r}) .
$$

Finally, we rely on the formal equivalence [8]

$$
E_{\mathrm{xc}, \lambda=1}[n] \equiv E_{\mathrm{xc}}[n]-T_{\mathrm{c}}[n]
$$

to extract a representation,

$$
E_{\mathrm{c}, \lambda=1}^{\mathrm{nl}}[n] \equiv E_{\mathrm{c}}^{\mathrm{nl}}[n]-T_{\mathrm{c}}^{\mathrm{nl}}[n],
$$

of the mutual plasmon electrodynamical coupling in the physical system $[1,3,4,6]$.

As implied in Eqs. (5) and (6), the code also gives us access to spatially resolved kinetic-correlation energies, $t_{\mathrm{c}}^{\mathrm{nl}}[n](\mathbf{r})$ and $t_{\mathrm{c}}[n](\mathbf{r})$, that are consistent with Eqs. (3) and (4) and with the standard resolution of $\mathrm{XC}$ energy contributions. Using PPACF, we can thus compute and discuss the nature of bindinginduced changes $\Delta t_{\mathrm{c}}(\mathbf{r}), \Delta t_{\mathrm{c}}^{\mathrm{nl}}(\mathbf{r})$, and $\Delta e_{\mathrm{c}}^{\mathrm{nl}}(\mathbf{r})-\Delta t_{\mathrm{c}}^{\mathrm{nl}}(\mathbf{r})$, in the spatially resolved descriptions. Our PPACF code can provide this analysis for most versions or variants of the vdW-DF method $[15,17,18,20,57-60]$. Here we work with the consistent-exchange vdW-DF-cx formulation [18,24], which can effectively be seen as a mean-value evaluation of the $\mathrm{ACF}$ [6].

We find that $\Delta t_{\mathrm{c}}(\mathbf{r})$ and $\Delta e_{\mathrm{c}}^{\mathrm{nl}}(\mathbf{r})$ both contain signatures of directed binding: the dominant binding contributions are channeled into pockets. Also, the signatures in $\Delta t_{\mathrm{c}}^{\mathrm{nl}}(\mathbf{r})$ and in $\Delta e_{\mathrm{c}}^{\mathrm{nl}}(\mathbf{r})$ typically mirror each other, up to a sign. This means that the concentration of vdW bonding is further enhanced in the contribution $\Delta e_{\mathrm{c}}^{\mathrm{nl}}(\mathbf{r})-\Delta t_{\mathrm{c}}^{\mathrm{nl}}(\mathbf{r})$ that characterizes the electrodynamical coupling mechanism behind the $\mathrm{vdW}$ attraction $[1,3,4,6]$.

Overall, our results show that there is an important kineticenergy nature of vdW binding and confirm that the density tails, rather than the atomic centers, play the decisive role in setting dispersion forces at binding separations [6,14,18,31,34-36,61-63]. Our results also suggest that there exists an orbital-like structure of dispersion binding, although much weaker than in chemical bonds and originating in different mechanisms $[4,6,16]$. This observation could be useful for qualitative discussions of the nature and variation in $\mathrm{vdW}$ forces in materials.

The rest of this paper is organized as follows. Section II details the coupling constant analysis of the vdW-DF method. Section III provides computational details. In Sec. IV, we document signatures of the vdW attraction in both noncovalent and covalent molecular binding. Section V contains a summary and discussion. The paper has one appendix. 


\section{THEORY}

A systematic theory characterization of the screened response in a homogeneous and weakly perturbed electron gas $[2,5,28,29,64-66]$ has led to the definition of a range of successful constraint-based functionals for the XC energy $E_{\mathrm{xc}}$ and broad use of DFT. We use $\hat{V}$ to denote the full electron-electron interaction. We consider the density changes $\delta n$ produced by an external field $\delta \Phi_{\text {ext }}$, and compute the electron-gas density response $\chi_{\lambda}=\delta n / \delta \Phi_{\text {ext }}$ as a function of the assumed coupling constant $\lambda$ for an adiabatic turn on of the many-particle interaction, $V_{\lambda}=\lambda V$. The exact $\mathrm{XC}$ energy is given by the $\mathrm{ACF}$,

$$
E_{\mathrm{xc}}=-\int_{0}^{\infty} \frac{d u}{2 \pi} \operatorname{Tr}\left\{\chi_{\lambda}(i u) V\right\}-E_{\text {self }},
$$

which links $\lambda$, the (complex) frequency $i u$, and spatial variations in the response function $\chi_{\lambda}$ to the XC energy. We use $\hat{n}(\mathbf{r})$ to denote the density operator, and the last term of Eq. (9) is the electron self-energy $E_{\text {self }}=\operatorname{Tr}\{\hat{n} V\} / 2$.

The exact XC energy can be recast as an electrostatic interaction $[2,28,29]$

$$
E_{\mathrm{xc}}=\frac{1}{2} \int_{\mathbf{r}} \int_{\mathbf{r}^{\prime}} \frac{n(\mathbf{r}) n_{\mathrm{xc}}\left(\mathbf{r} ; \mathbf{r}^{\prime}\right)}{\left|\mathbf{r}-\mathbf{r}^{\prime}\right|}
$$

between the electrons and associated, so-called, $\mathrm{XC}$ holes $n_{\mathrm{xc}}\left(\mathbf{r} ; \mathbf{r}^{\prime}\right)$. The XC hole reflects a $\lambda$ average of the response $\chi_{\lambda}$. An emphasis on the assumed plasmon-nature of the electron response, a reliance on formal many-body perturbation theory, and the imposing of additional physics constraints, such as charge conservation of the $\mathrm{XC}$ hole, has led to formulations of LDA [67,68], of the PBE and PBEsol versions of GGAs $[21,22]$, and of the vdW-DF method [6,15-17,20,24].

At any given coupling constant $\lambda$, the response function defines an approximation for the exchange-correlation hole

$$
n_{\mathrm{xc}, \lambda}\left(\mathbf{r}, \mathbf{r}^{\prime}=\mathbf{r}+\mathbf{w}\right)=-\frac{2}{n(\mathbf{r})} \int_{0}^{\infty} \frac{d u}{2 \pi} \chi_{\lambda}\left(\mathbf{r}, \mathbf{r}^{\prime} ; i u\right)-\delta(\mathbf{w}) .
$$

The actual $\mathrm{XC}$ hole then emerges simply as an average,

$$
n_{\mathrm{xc}}=\int_{0}^{1} n_{\mathrm{xc}, \lambda} d \lambda
$$

Using Eq. (11), it is meaningful to define and discuss also the coupling-constant dependence of the XC functional:

$$
E_{\mathrm{xc}, \lambda} \equiv \frac{1}{2} \int_{\mathbf{r}} \int_{\mathbf{r}^{\prime}} \frac{n(\mathbf{r}) n_{\mathrm{xc}, \lambda}\left(\mathbf{r}, \mathbf{r}^{\prime}\right)}{\left|\mathbf{r}-\mathbf{r}^{\prime}\right|} .
$$

Same as for the holes, the actual functional, Eq. (10), is given by an average over $0<\lambda<1$,

$$
E_{\mathrm{xc}}[n]=\int_{0}^{1} d \lambda E_{\mathrm{xc}, \lambda}[n] .
$$

The behavior of $E_{\mathrm{xc}, \lambda}$ is exclusively set by exchange effects at $\lambda=0$. This follows because exchange reflects an independent-particle behavior and, unlike correlation, it is independent of $\lambda$. At the other physical limit, the plasmon character can be expected to dominate in the response. One therefore also expects that $E_{\mathrm{xc}, \lambda}$ becomes accurate at $\lambda \rightarrow 1$ if Eq. (13) reflects a plasmon-based analysis of electron response, for example, as used in the early LDA formulations [29,67], in the constraint-based GGAs [21,22], and in vdW-DF-cx [18].

It is instructive to split the $\mathrm{XC}$ hole into exchange and correlation components,

$$
n_{\mathrm{xc}, \lambda}\left(\mathbf{r}, \mathbf{r}^{\prime}\right)=n_{\mathrm{x}}\left(\mathbf{r}, \mathbf{r}^{\prime}\right)+n_{\mathrm{c}, \lambda}\left(\mathbf{r}, \mathbf{r}^{\prime}\right),
$$

and to define (at every $\lambda$ ) a spatially resolved correlation term:

$$
e_{\mathrm{c}, \lambda}[n](\mathbf{r})=\frac{n(\mathbf{r})}{2} \int_{\mathbf{r}^{\prime}} \frac{n_{\mathrm{c}, \lambda}\left(\mathbf{r}, \mathbf{r}^{\prime}\right)}{\left|\mathbf{r}-\mathbf{r}^{\prime}\right|} .
$$

This term provides a mapping of the total correlation effects at $\lambda$ :

$$
E_{\mathrm{c}, \lambda}[n]=\int_{\mathbf{r}} e_{\mathrm{c}, \lambda}(\mathbf{r}) .
$$

Also, there exists a coupling-constant scaling analysis for LDA correlation $[10,11,54] E_{\mathrm{c}, \lambda}^{\mathrm{LDA}}$ with spatial resolution

$$
E_{\mathrm{c}, \lambda}^{\mathrm{LDA}}[n]=\int_{\mathbf{r}} e_{\mathrm{c}, \lambda}^{\mathrm{LDA}}[n](\mathbf{r}) .
$$

Accordingly, we isolate a spatially resolved nonlocalcorrelation energy

$$
e_{\mathrm{c}, \lambda}^{\mathrm{nl}}[n](\mathbf{r})=e_{\mathrm{c}, \lambda}[n](\mathbf{r})-e_{\mathrm{c}, \lambda}^{\mathrm{LDA}}[n](\mathbf{r}),
$$

corresponding to the coupling-constant scaling of the total nonlocal-correlation energy:

$$
E_{\mathrm{c}, \lambda}^{\mathrm{nl}}[n]=\int_{\mathbf{r}} e_{\mathrm{c}, \lambda}^{\mathrm{nl}}[n](\mathbf{r}) .
$$

Equation (3) is the coupling-constant integral of $e_{\mathrm{c}, \lambda}^{\mathrm{nl}}[n](\mathbf{r})$. To map the electrodynamical-coupling nature of $\mathrm{vdW}$ attraction, we seek to compute the binding-induced changes $\Delta e_{\mathrm{c}, \lambda=1}^{\mathrm{nl}}[n](\mathbf{r})$.

\section{A. Density scaling in the exact XC energy}

Coupling-constant scaling analysis [8] is a natural tool for exploring the nature of both exchange-based GGAs $[11,56]$ and of vdW-DF-cx. For any given solution density $n(\mathbf{r})$, we define a rescaled density

$$
n(\mathbf{r}) \rightarrow n_{1 / \lambda}(\mathbf{r}) \equiv n(\mathbf{r} / \lambda) / \lambda^{3},
$$

and resolve Eq. (14) into $\lambda$-specific contributions using the exact result $[8,11,56]$

$$
E_{\mathrm{xc}, \lambda}[n]=\frac{d}{d \lambda}\left\{\lambda^{2} E_{\mathrm{xc}}\left[n_{1 / \lambda}\right]\right\} .
$$

Since there is no $\lambda$ dependence for exchange, we can recast Eq. (22) using the correlation-energy density:

$$
e_{\mathrm{c}, \lambda}[n](\mathbf{r})=\frac{d}{d \lambda}\left\{\lambda^{2} e_{\mathrm{c}}\left[n_{1 / \lambda}\right](\mathbf{r})\right\} .
$$

The scaling results for $E_{\mathrm{xc}, \lambda}[n]$ and $e_{\mathrm{c}, \lambda}[n]$ can be directly applied to individual components of the XC functional (as they are linear in the functional expression).

The scaling results, Eqs. (22) and (23), reflect properties of the $\chi_{\lambda}$ approximations that are implicitly made in crafting the PBE and vdW-DF-cx functionals. The existence of a well-understood coupling-constant scaling has been used to rationalize the formulation of the PBE0 hybrid [69] based on 
PBE $[11,56]$. Noting that a similar rationale exists for the coupling constant scaling of vdW-DF-cx, some of us have recently motivated the introduction of correspondingly defined vdW-DF hybrids, including vdW-DF-cx0, which replace the vdW-DF-cx exchange component with a fraction of the Fock exchange [70].

The scaling results, Eqs. (22) and (23), follow from an analysis of the many-particle wave function ground-state solution $\Psi_{n}^{\min , \lambda}$ corresponding to a specific density $n$ and a specific strength $\lambda V$ of the electron-electron interaction. The detailed arguments are given elsewhere. For completeness, we include a renormalization-type argument for this observation in Appendix. Here, we simply note that the wave functions solving the Hamiltonian $\hat{H}=\hat{T}+\lambda V+V_{\text {ext }}$ themselves scale according to

$$
\Psi_{n}^{\min , \lambda}\left(\mathbf{r}_{1}, \ldots, \mathbf{r}_{N}\right)=\lambda^{3 N / 2} \Psi_{n_{1 / \lambda}}\left(\lambda \mathbf{r}_{1}, \ldots, \lambda \mathbf{r}_{N}\right),
$$

and that this formal equivalence is sufficient to establish the $\lambda$ scaling $[8,11]$.

\section{B. Access to the kinetic-correlation energy}

Below, we drop explicit references to the density functional nature when working with spatially resolved energy contributions such as $e_{\mathrm{c}, \lambda}^{\mathrm{nl}}(\mathbf{r})$ and $e_{\mathrm{c}}^{\mathrm{nl}}(\mathbf{r})$, except when specifically needed for the discussion.

In the KS scheme [7], we nominally focus on computing the so-called KS kinetic energy ${ }^{2}$ from single-particle expectation values,

$$
\begin{gathered}
\left\langle\phi_{i}|\hat{T}| \phi_{i}\right\rangle=\int_{\mathbf{r}} \tilde{t}_{i}(\mathbf{r}), \\
\tilde{t}_{i}(\mathbf{r})=-\frac{1}{2} \phi_{i}^{*}(\mathbf{r}) \nabla^{2} \phi_{i}(\mathbf{r}),
\end{gathered}
$$

for occupied orbitals $\phi_{i}(\mathbf{r})$. As in the QUANTUM ESPRESSO package [71], we compute the KS kinetic energy as a spatial integration

$$
T_{\mathrm{KS}}[n]=\int_{\mathbf{r}} t_{\mathrm{KS}}(\mathbf{r}),
$$

over positive definite contributions,

$$
t_{\mathrm{KS}}(\mathbf{r})=\frac{1}{2} \sum_{i}^{\mathrm{occ}}\left|\nabla \phi_{i}(\mathbf{r})\right|^{2},
$$

defined by the set of occupied orbitals. This representation of the KS kinetic energy is simply related to the summation $\tilde{t}_{\text {occ }}(\mathbf{r}) \equiv \Sigma_{i}^{\text {occ }} \tilde{t}_{i}(\mathbf{r})$ over single-particle contributions, Eq. (26). The descriptions differ only in the inclusion of a Poisson-type term

$$
t_{\mathrm{KS}}(\mathbf{r})=\frac{1}{4} \nabla^{2} n(\mathbf{r})+\operatorname{Re}\left\{\tilde{t}_{\mathrm{occ}}(\mathbf{r})\right\}
$$

and give the same total KS kinetic energy, Eq. (27), upon spatial integration.

We typically compute DFT energies $E_{\mathrm{DFT}}^{\mathrm{A} / \mathrm{B} / \mathrm{AB}}$ of combined systems "AB" and of the relevant fragments, "A" or "B,"

\footnotetext{
${ }^{2}$ In discussions of DFT, $T_{\mathrm{KS}}$ is sometimes called the single-particle kinetic energy. We prefer the Kohn-Sham label as $\hat{T}$ is always a singleparticle operator.
}

to understand binding $\Delta E_{\mathrm{DFT}}=E_{\mathrm{DFT}}^{\mathrm{A}}+E_{\mathrm{DFT}}^{\mathrm{B}}-E_{\mathrm{DFT}}^{\mathrm{AB}}$ (with suitable adjustments in the case of related problems such as material cohesion). The mean-field electrostatic energy among electrons, that is, the Hartree term

$$
U[n]=\frac{1}{2} \int_{\mathbf{r}} \int_{\mathbf{r}^{\prime}} \frac{n(\mathbf{r}) n\left(\mathbf{r}^{\prime}\right)}{\left|\mathbf{r}-\mathbf{r}^{\prime}\right|},
$$

is one important contribution as it approximates $\langle\hat{V}\rangle$. For analysis, we track binding-induced changes like

$$
\begin{gathered}
\Delta U \equiv U^{\mathrm{A}}+U^{\mathrm{B}}-U^{\mathrm{AB}}, \\
\Delta T_{\mathrm{KS}} \equiv T_{\mathrm{KS}}^{\mathrm{A}}+T_{\mathrm{KS}}^{\mathrm{B}}-T_{\mathrm{KS}}^{\mathrm{AB}} \\
\Delta T_{\mathrm{c}}^{\mathrm{nl}} \equiv T_{\mathrm{c}}^{\mathrm{nl}, \mathrm{A}}+T_{\mathrm{c}}^{\mathrm{nl}, \mathrm{B}}-T_{\mathrm{c}}^{\mathrm{nl}, \mathrm{AB}} .
\end{gathered}
$$

We also track the corresponding expressions for bindinginduced changes in, for example, the total nonlocal-correlation term $\Delta E_{\mathrm{c}}^{\mathrm{nl}}$. In our discussion, we call such differences binding contributions. ${ }^{3}$

Computational results for the binding-induced changes in the KS kinetic energy $\Delta T_{\mathrm{KS}}$ and in the mean-field electrostatic energy $\Delta U[n]$ often suffice for a characterization of covalent bonds in molecules and materials [72]. This is because the combination allows us to characterize and understand orbital hybridization [72-74]. However, for noncovalent bonds, we have to look further than changes in $T_{\mathrm{KS}}$. One can generally sort chemical bonds from knowledge of the average orbital energy [75]. The average orbital energy is a measure that will, in principle, reflect all correlation effects, including those that are manifested in the kinetic energy.

A formal analysis of the DFT variational scheme [8] shows that

$$
T_{\mathrm{c}}[n]=-E_{c}[n]+\left[\frac{\partial E_{c}\left[n_{\alpha}\right]}{\partial \alpha}\right]_{\alpha=1},
$$

where $\alpha \equiv 1 / \lambda$, Refs. [8,11]. Using the density-scaling analysis, it immediately follows that

$$
T_{\mathrm{c}}[n]=E_{\mathrm{c}}[n]-E_{\mathrm{c}, \lambda=1}[n]=E_{\mathrm{xc}}[n]-E_{\mathrm{xc}, \lambda=1}[n] .
$$

A similar equation connects $T_{\mathrm{c}}^{\mathrm{nl}}[n]$ and $E_{\mathrm{c}}^{\mathrm{nl}}[n]$. For any given system (solution density $n$ ), we use numerical differentiation to compute $E_{\mathrm{c}, \lambda}[n]$ and $E_{\mathrm{c}, \lambda}^{\mathrm{nl}}[n]$ from Eq. (22), and $T_{\mathrm{c}}[n]$ and $T_{\mathrm{c}}^{\mathrm{nl}}[n]$ from Eq. (34).

The electron-electron interaction effects in the physical systems are now formally available for computation (in the approximations that define $\left.E_{\mathrm{xc}}[n]\right)$. In particular, we can study the electrodynamical coupling among plasmons $[1,3,4,6]$ at $\lambda=1$ since $E_{\mathrm{c}, \lambda=1}^{\mathrm{nl}}[n]$ is available via Eq. (35). This value $E_{\mathrm{c}, \lambda=1}^{\mathrm{nl}}[n]$ is the nonlocal-correlation part of $E_{\mathrm{xc}, \lambda=1}[n]$, which by definition, is given by a contour integral of the response

\footnotetext{
${ }^{3}$ The wording "binding contribution" is used to describe any component of the molecular binding even if, for example, $\Delta T_{\mathrm{c}}(\mathbf{r})$ is negative. Similarly, we use the word "spatially resolved binding contributions" to describe binding-induced changes in the spatial variation of, for example, $\mathrm{XC}$ energy terms, like $\Delta e_{\mathrm{c}}^{\mathrm{nl}}(\mathbf{r})$; Again this term is used without regards to the sign of the integrated values.
} 


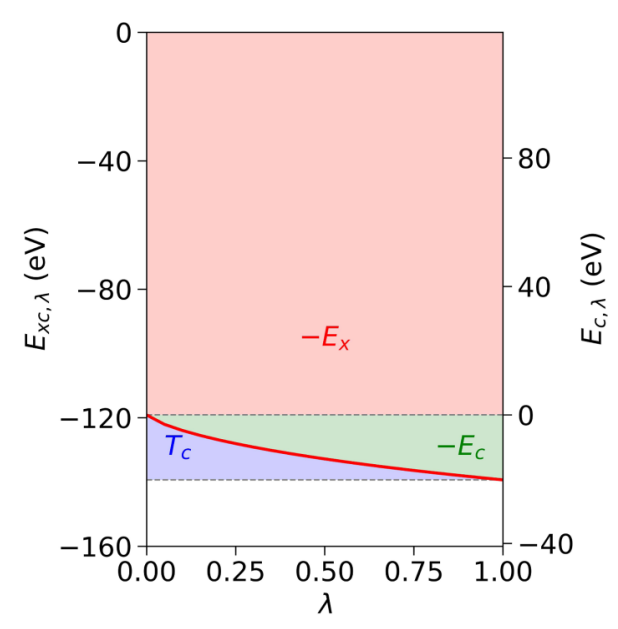

FIG. 1. Coupling-constant scaling of the vdW-DF-cx exchange and correlation contributions to the total energy of the $\mathrm{N}_{2}$ molecule. The solid curve (upper dashed line) shows variation in total XC term $E_{\mathrm{xc}, \lambda}[n]$ (lack of variation in exchange term $E_{\mathrm{x}, \lambda}[n]$ ) for the $\mathrm{N}_{2}$ solution electron density $n(\mathbf{r})$. The area of the green (red) regions is minus the total correlation (exchange) energy, while the area of the blue region is the so-called kinetic-correlation energy, that is, the kinetic-energy part of correlation, $T_{\mathrm{c}}[n]$.

$\chi_{\lambda=1}$ evaluated at full electron-electron interaction strength, Eqs. (11) and (13).

We note in passing that $E_{\mathrm{c}, \lambda=1}^{\mathrm{nl}}[n]$ is also the nonlocalcorrelation part of the electron-electron interaction expectation value

$E_{\mathrm{c}, \lambda=1}^{\mathrm{nl}}[n]=\langle\hat{V}\rangle_{\mathrm{c}}^{\mathrm{nl}} \equiv\langle\hat{V}\rangle-U[n]-E_{\mathrm{x}}[n]-E_{\mathrm{c}, \lambda=1}^{\mathrm{LDA}}[n]$.

Since the $\mathrm{XC}$ energy functional is defined $E_{\mathrm{xc}}=\langle\hat{V}+\hat{T}\rangle-$ $T_{\mathrm{KS}}-U[n]$ we can use Eq. (35) for the formal identification

$$
E_{\mathrm{xc}, \lambda=1}[n]=\langle\hat{V}\rangle-U[n] .
$$

The formal equivalence (36) follows by subtracting the LDA and gradient-corrected exchange components.

Figure 1 shows (computed results for) the coupling constant scaling for the XC contribution (solid red curve) to the total energy of the $\mathrm{N}_{2}$ molecules. The specific scaling results are here provided for vdW-DF-cx (using the formal derivation of the scaling for $E_{\mathrm{c}}^{\mathrm{nl}}$ detailed in the following section). However, the behavior is generic and thus similar to what has previously been reported and discussed for PBE $[11,56]$.

We note that the exchange and correlation components, $E_{\mathrm{x}}$ and $E_{\mathrm{c}}$, used for DFT calculations in the KS scheme, are integrals of the indicated $\lambda$ variations. The exchange value traces a horizontal line (dotted curve separating red and green areas) in Fig. 1. In contrast, the correlation begins at zero but changes to a significant magnitude at $\lambda=1$. It is straightforward to verify [11] that the area of the green region is minus the functional approximation for $E_{\mathrm{c}}$. Importantly, we can immediately extract the corresponding kinetic-correlation energy $T_{\mathrm{c}}$ using Eq. (35), that is, as the area of the blue region below the $E_{\mathrm{xc}, \lambda}$ variation but above the value of the $\lambda \rightarrow 1$ limit.

\section{Coupling-constant scaling and kinetic-correlation energy in vdW-DF-cx}

To compute the kinetic-energy component of vdW binding, we need only to consider the density scaling for the correlation parts, namely $E_{c}=E_{c}^{\mathrm{LDA}}+E_{c}^{\mathrm{nl}}$. Moreover, the coupling constant scaling for the LDA part, $E_{c}^{\mathrm{LDA}}$, has previously been discussed, as it is part of the GGA characterization [8-11,54-56,76,77].

To explore the coupling-constant scaling of $E_{\mathrm{c}}^{\mathrm{nl}}$, we first summarize the vdW-DF formulation of this nonlocalcorrelation energy. Any semilocal XC density functional can be characterized by a local energy-per-particle density

$$
E_{\mathrm{xc}}[n]=\int_{\mathbf{r}} n(\mathbf{r}) \epsilon_{\mathrm{xc}}[n](\mathbf{r}),
$$

where $\epsilon_{\mathrm{xc}}[n](\mathbf{r})$ is a function of just the local density $n(\mathbf{r})$ and the scaled density gradient $s(\mathbf{r})$. We further split $\epsilon_{\mathrm{xc}}[n](\mathbf{r})$ into exchange and correlation components, $\epsilon_{\mathrm{x}}[n](\mathbf{r})$ and $\epsilon_{\mathrm{c}}[n](\mathbf{r})$. The local variation in the inverse length scale $q_{0}$ for the plasmon-pole description can then be expressed as $[15,16]$

$$
\begin{gathered}
q_{0}(\mathbf{r})=q_{0 x}(\mathbf{r})+q_{0 c}(\mathbf{r}), \\
q_{0 x(c)}(\mathbf{r})=\frac{\varepsilon_{\mathrm{x}(\mathrm{c})}^{\mathrm{in}}(\mathbf{r})}{\varepsilon_{x}^{\mathrm{LDA}}(\mathbf{r})} k_{F}(\mathbf{r}) .
\end{gathered}
$$

Here, $k_{F}=\left(3 \pi^{2} n\right)^{1 / 3}$ denotes the local value of the Fermi wave vector and $\varepsilon_{x}^{\mathrm{LDA}}=-3 k_{F} / 4 \pi$ is the energy-per-particle density in LDA exchange. The nonlocal-correlation energy [15],

$$
E_{\mathrm{c}}^{\mathrm{nl}}=\frac{1}{2} \int_{\mathbf{r}} \int_{\mathbf{r}^{\prime}} n(\mathbf{r}) \phi\left(\mathbf{r}, \mathbf{r}^{\prime}\right) n\left(\mathbf{r}^{\prime}\right),
$$

is computed using a universal-kernel formulation $[15,16,27,32]$,

$$
\begin{gathered}
\phi\left(\mathbf{r}, \mathbf{r}^{\prime}\right)=\Phi_{0}\left(d, d^{\prime}\right), \\
d\left(\mathbf{r}, \mathbf{r}^{\prime}\right)=\left|\mathbf{r}-\mathbf{r}^{\prime}\right| q_{0}(\mathbf{r}), \\
d^{\prime}\left(\mathbf{r}, \mathbf{r}^{\prime}\right)=\left|\mathbf{r}-\mathbf{r}^{\prime}\right| q_{0}\left(\mathbf{r}^{\prime}\right) .
\end{gathered}
$$

The universal kernel $\Phi_{0}\left(d, d^{\prime}\right)$ is tabulated and permits an efficient numerical evaluation through fast-Fourier transforms [27]. The generalization to scaling in spin-polarized cases is also completely specified, since it amounts to a simple rescaling of the inverse length scale $q_{0}$, Ref. [19].

For scaling analysis (and coding), it is convenient to introduce $\tilde{\mathbf{r}} \equiv \mathbf{r} / \lambda$ as a short hand for the coordinate scaling and to represent the density variation in terms of $r_{s}(\mathbf{r})=$ $(3 / 4 \pi n(\mathbf{r}))^{1 / 3}$. The local values of the scaled density gradient are $s(\mathbf{r})=|\nabla n| /\left(2 k_{F}(\mathbf{r}) n(\mathbf{r})\right)$. The density scaling $n(\mathbf{r}) \rightarrow$ $n_{1 / \lambda}(\mathbf{r})=n(\tilde{\mathbf{r}}) / \lambda^{3}$ leaves $s$ unchanged and the effect amounts to computing the changes in Fermi vector and in the LDA correlation components. This is done in terms of the corresponding scaling $r_{s}(\mathbf{r}) \rightarrow \lambda r_{s}(\tilde{\mathbf{r}})$.

Using $F_{x}(s)$ to denote the exchange-enhancement factor of $E_{\mathrm{xc}}^{\mathrm{in}}$, the overall scaling of the inverse length scale can be 
expressed $q_{0}\left[n_{1 / \lambda}(\mathbf{r})\right]=q_{0}^{\lambda}(\tilde{\mathbf{r}})$, where

$$
q_{0}^{\lambda}(\tilde{\mathbf{r}})=F_{x}(s(\tilde{\mathbf{r}})) \frac{k_{F}(\tilde{\mathbf{r}})}{\lambda}-\frac{4 \pi}{3} \varepsilon_{\mathrm{c}}^{\mathrm{LDA}}\left(\lambda r_{s}(\tilde{\mathbf{r}})\right) .
$$

Introducing also

$$
\begin{aligned}
\tilde{d}_{\lambda} & \equiv q_{0}^{\lambda}(\tilde{\mathbf{r}})\left(\lambda\left|\tilde{\mathbf{r}}-\tilde{\mathbf{r}}^{\prime}\right|\right), \\
\tilde{d}_{\lambda}^{\prime} & \equiv q_{0}^{\lambda}\left(\tilde{\tilde{\mathbf{r}}^{\prime}}\right)\left(\lambda\left|\tilde{\mathbf{r}}-\tilde{\mathbf{r}}^{\prime}\right|\right),
\end{aligned}
$$

we can compute

$$
E_{\mathrm{c}}^{\mathrm{nl}}\left[n_{1 / \lambda}\right]=\int_{\tilde{\mathbf{r}}} \int_{\tilde{\mathbf{r}}^{\prime}} n(\tilde{\mathbf{r}}) \Phi_{0}\left(\tilde{d}_{\lambda}, \tilde{d}_{\lambda}^{\prime}\right) n\left(\tilde{\mathbf{r}}^{\prime}\right) .
$$

We complete the scaling analysis of $E_{\mathrm{c}}^{\mathrm{nl}}$ via Eq. (22) and of $e_{\mathrm{c}}^{\mathrm{nl}}(\mathbf{r})$ via Eq. (23). Specifically, we express the scaling of the nonlocal-correlation energy density

$$
e_{\mathrm{c}, \lambda}^{\mathrm{nl}}(\mathbf{r})=\frac{d}{d \lambda}\left\{\lambda^{2} e_{\mathrm{c}}^{\mathrm{nl}}\left[\lambda r_{s}(\tilde{\mathbf{r}})\right]\right\} .
$$

For the numerical evaluation, we adopted the scheme proposed by Román-Pérez and Soler [27] (as implemented in QUANTUM ESPRESSO) to calculate $e_{\mathrm{c}}^{\mathrm{nl}}\left[\lambda r_{s}(\tilde{\mathbf{r}})\right]$; the calculation is similar to the calculation of $e_{\mathrm{c}}^{\mathrm{nl}}(\mathbf{r})$ in Ref. [30]. We note that $e_{\mathrm{c}, \lambda=1}(\mathbf{r})$ provides a spatial mapping of all nonlocal-correlation effects that exist in the fully interacting system, as a direct implication of Eq. (35).

Moreover, as part of this $e_{\mathrm{c}, \lambda=1}(\mathbf{r})$ characterization, we can now compute the spatially resolved kinetic-correlation energy $t_{\mathrm{c}}(\mathbf{r})$ and nonlocal-kinetic-correlation energy $t_{\mathrm{c}}^{\mathrm{nl}}(\mathbf{r})$. First, we simply add the known [8-10,54] coupling constant scaling of the LDA correlation-energy density $e_{\mathrm{c}, \lambda}^{\mathrm{LDA}}$, entering in Eq. (18):

$$
e_{\mathrm{c}, \lambda}(\mathbf{r})=e_{\mathrm{c}, \lambda}^{\mathrm{LDA}}(\mathbf{r})+e_{\mathrm{c}, \lambda}^{\mathrm{nl}}(\mathbf{r}) .
$$

Next, we adapt Eq. (34) to descriptions of energy densities

$$
\begin{gathered}
t_{\mathrm{c}}(\mathbf{r})=-e_{\mathrm{c}}(\mathbf{r})+\left[\frac{\partial e_{\mathrm{c}}\left[r_{s}(\tilde{\mathbf{r}}) / \alpha\right]}{\partial \alpha}\right]_{\alpha=1}, \\
t_{\mathrm{c}}^{\mathrm{nl}}(\mathbf{r})=-e_{\mathrm{c}}^{\mathrm{nl}}(\mathbf{r})+\left[\frac{\partial e_{\mathrm{c}}^{\mathrm{nl}}\left[r_{s}(\tilde{\mathbf{r}}) / \alpha\right]}{\partial \alpha}\right]_{\alpha=1},
\end{gathered}
$$

and evaluate the derivatives numerically.

Figure 2 documents the coupling-constant scaling of $E_{\mathrm{c}, \lambda}^{\mathrm{nl}}$ and explains an approximately linear variation. The top panel shows the coupling-constant scaling for the ratio $q_{0 c} / q_{0 x}$ for typical contributions to the binding of an $\mathrm{N}_{2}$ molecule. Specifically, starting from the known $q_{0 x}(n(\mathbf{r}))$ and $q_{0 c}(n(\mathbf{r}))$ values, the panel traces the variation in the $\mathrm{XC}$ components of $q_{0}\left(n_{1 / \lambda}(\mathbf{r})\right)$ for conditions that roughly correspond to the binding region of $\mathrm{N}_{2}$ (red curves) and to electron density tails of atoms and molecules (blue curves).

The middle panel of Fig. 2 shows the coupling-constant scaling of $E_{\mathrm{c}}^{\mathrm{nl}}\left[n_{1 / \lambda}\right]$ (solid curve) for the density of the $\mathrm{N}_{2}$ molecule. We note that the scaling in $q_{0 x}$ is exactly offset by the $\lambda$ scaling of coordinates in Eqs. (46) and (47). Thus if we assume that the scaling of $q_{0}=q_{0 x}+q_{0 c}$ is set by the scaling in $q_{0 x}$, there would be no $\lambda$ dependence in the $E_{\mathrm{c}}^{\mathrm{nl}}$ kernel arguments, $d=q_{0}(\mathbf{r})\left|\mathbf{r}-\mathbf{r}^{\prime}\right|$ and $d^{\prime}=q_{0}\left(\mathbf{r}^{\prime}\right)\left|\mathbf{r}-\mathbf{r}^{\prime}\right|$. In this type of approximations, there is then no scaling in the corresponding approximations for $E_{\mathrm{c}}^{\mathrm{nl}}\left[n_{1 / \lambda}\right]$.

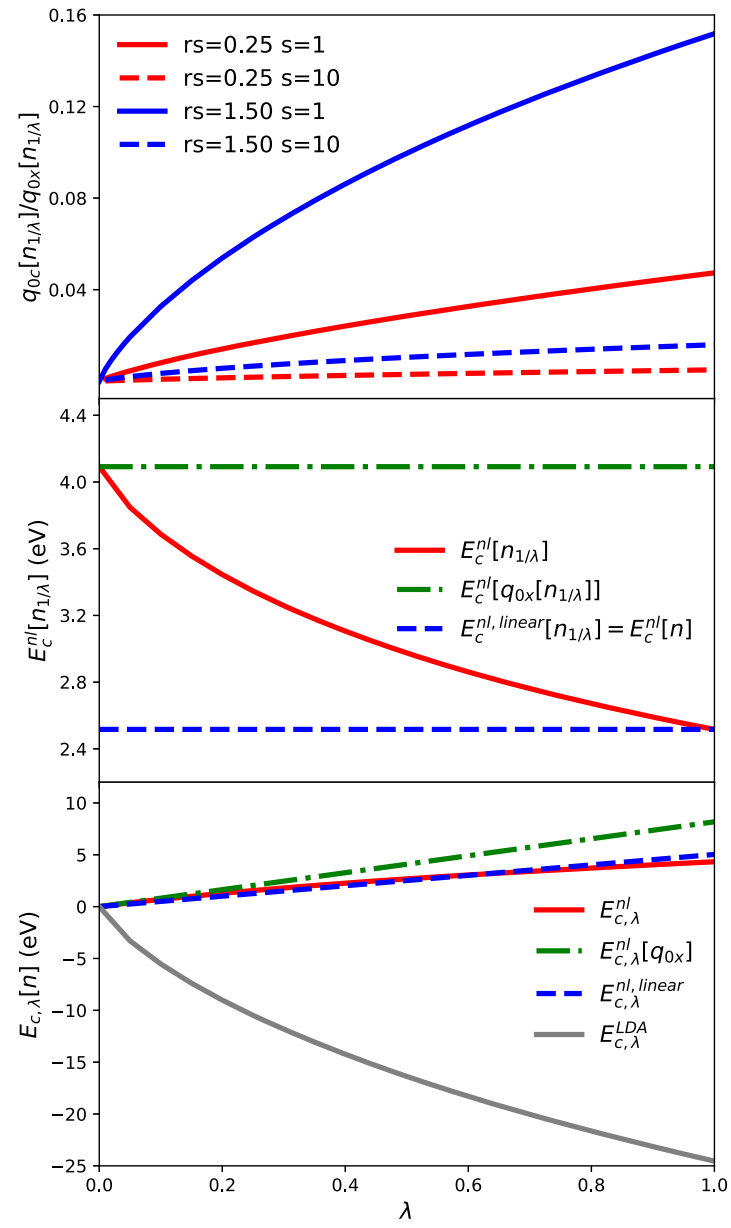

FIG. 2. Coupling-constant scaling of the nonlocal correlation energy functional $E_{\mathrm{c}, \lambda}^{\mathrm{nl}}[n]$ at various density conditions typical of the $\mathrm{N}_{2}$ molecule. The binding is thus analyzed in terms of values for the inverse length scales, $q_{0}=q_{0 x}+q_{0 c}$, that specify the vdWDF plasmon model. The top panel shows the scaling in the ratio $q_{0 c} / q_{0 x}$ at conditions typical for the interatom binding region of the $\mathrm{N}_{2}$ molecules (for the nonbinding regions outside each atom), red (blue) solid curves. The set of dashed curves shows a corresponding characterization for density tails. The middle panel shows the resulting scaling of $E_{\mathrm{c}}^{\mathrm{nl}}\left[n_{1 / \lambda}\right]$ (solid curve) with limits discussed in the text. The bottom panel contrasts the resulting scaling in $E_{\mathrm{c}, \lambda}^{\mathrm{nl}}[n]$ (solid red curve) against that of LDA correlation (solid grey curve), and as obtained in two approximations.

The middle panel furthermore shows two potentially relevant such approximations motivated by the analysis of the typical variations in the $q_{0 c} / q_{0 x}$ ratio. The first assumes that we can ignore the influence of the correlation part $q_{0 c}$ completely (giving the green dashed-dotted line); the second assumes that the ratio $q_{0 c} / q_{0 x}$ can at any given point $\mathbf{r}$ be taken as fixed at the $\lambda=1$ value (giving the blue dashed curve). The second choice effectively amounts to simply setting $E_{\mathrm{c}}^{\mathrm{nl}, \text { linear }}\left[n_{1 / \lambda}\right] \equiv E_{\mathrm{c}}^{\mathrm{nl}}[n]$. Neither of them is a good description for Eq. (48). On the other hand, we add a $\lambda^{2}$ weight on $E_{\mathrm{c}}^{\mathrm{nl}}\left[n_{1 / \lambda}\right]$ when computing $E_{\mathrm{xc}, \lambda}$, Eq. (22). We label the second approximation as "linear" since it leads to $E_{\mathrm{c}, \lambda}^{\mathrm{nl}} \approx \lambda E_{\mathrm{c}}^{\mathrm{nl}}[n]$ and this is sometimes an acceptable approximation.

The bottom panel of Fig. 2 contrasts the resulting scaling of the ACF integrand for the nonlocal correlation contribution 
$E_{\mathrm{c}, \lambda}^{\mathrm{nl}}[n]$ against that of the LDA correlation $E_{\mathrm{c}}^{\mathrm{LDA}}[n]$. The panel also shows (green and blue dashed curves) the scaling that results by inserting either of the approximations discussed in the middle panel into Eq. (22). Interestingly, the scaling relevant for the $\mathrm{N}_{2}$-molecule total energy is found well approximated by using $E_{\mathrm{c}}^{\mathrm{nl} \text {,linear }}\left[n_{1 / \lambda}\right] \equiv E_{\mathrm{c}}^{\mathrm{nl}}[n]$. The nonlocal-correlation part of the kinetic energy is just minus the total nonlocal-correlation binding contribution $\Delta E_{\mathrm{c}}^{\mathrm{nl}}$ in such special cases.

\section{COMPUTATIONAL DETAILS}

We focus our discussion and mapping of many-body physics effects on results obtained using the vdW-DF-cx version [18]. In vdW-DF-cx, the total exchange component in Eq. (2) is picked so that the binding contributions from $\delta E_{\mathrm{x}}^{0}$ can generally be ignored $[6,18]$.

The vdW-DF-cx version performs well, on par with or better than PBE, for characterizations of many bulk, surface, and interface properties [20,24,78-86]. The vdW-DF-cx version has proven itself useful also in the description of binding and function of layered materials, at surfaces, and of molecules [70,87-105].

Our calculations are based on the plane-wave QUANTUM ESPRESSO package [71], which already has the consistent exchange vdW-DF-cx version [18] as well as the rigorous spin extension of the vdW-DF method [16]. Core electrons are represented by Troullier-Martins type normal-conserving pseudo potentials using an 80-Ry wave-function cutoff.

This paper also introduces a post-processing ACF-analysis code, termed PPACF, which tracks the system-specific coupling constant variation in $E_{\mathrm{xc}}[n]$ (for standard GGA and vdW-DF versions). The code adapts the post-processing components of the QUANTUM ESPRESSO package [71], into which PPACF will also be released.

The PPACF code takes as input the set of QUANTUM ESPRESSO solution files (available after completion of the DFT calculations). It outputs the coupling-constant scaling analysis and the spatial variation in the kinetic-correlation energy density. For convenience, it also outputs the spatial variation in the set of $\mathrm{XC}$ components.

Our numerical analysis is based on comparing bindingenergy contributions for the various components of the total DFT description. To discuss the binding "AB" of fragments " $\mathrm{A}$ " and "B," the PPACF code outputs the spatial variation in all $\mathrm{XC}$ components. The code furthermore uses the coupling-constant scaling in the spatially resolved correlation terms, $e_{\mathrm{c}}$ and $e_{\mathrm{c}}^{\mathrm{nl}}$, to numerically determine and output (for any given fragment and for the combined system) the spatial variation in $e_{\mathrm{c}, \lambda=1}$ and $e_{\mathrm{c}, \lambda=1}^{\mathrm{nl}}$ as well as in $t_{\mathrm{c}, \lambda=1}$ and $t_{\mathrm{c}, \lambda=1}^{\mathrm{nl}}$. We then obtain, for the (spatially resolved) binding-energy contributions,

$$
\begin{gathered}
\Delta e_{\mathrm{c}, \lambda=1}=e_{\mathrm{c}, \lambda=1}^{\mathrm{A}}+e_{\mathrm{c}, \lambda=1}^{\mathrm{B}}-e_{\mathrm{c}, \lambda=1}^{\mathrm{AB}}, \\
\Delta e_{\mathrm{c}, \lambda=1}^{\mathrm{nl}}=e_{\mathrm{c}, \lambda=1}^{\mathrm{A}, \mathrm{nl}}+e_{\mathrm{c}, \lambda=1}^{\mathrm{B}, \mathrm{nl}}-e_{\mathrm{c}, \lambda=1}^{\mathrm{AB}, \mathrm{nl}}, \\
\Delta t_{\mathrm{c}, \lambda=1}=t_{\mathrm{c}, \lambda=1}^{\mathrm{A}}+t_{\mathrm{c}, \lambda=1}^{\mathrm{B}}-t_{\mathrm{c}, \lambda=1}^{\mathrm{AB}}, \\
\Delta t_{\mathrm{c}, \lambda=1}^{\mathrm{nl}}=t_{\mathrm{c}, \lambda=1}^{\mathrm{A}, \mathrm{nl}}+t_{\mathrm{c}, \lambda=1}^{\mathrm{B}, \mathrm{nl}}-t_{\mathrm{c}, \lambda=1}^{\mathrm{AB}, \mathrm{nl}},
\end{gathered}
$$

from simple numerical subtractions.
For completeness, the PPACF code outputs the spatial variation in KS kinetic energy $t_{\mathrm{KS}}(\mathbf{r})$ and in a spatially resolved measure of the full kinetic energy

$$
t_{\mathrm{tot}}(\mathbf{r}) \equiv t_{\mathrm{KS}}(\mathbf{r})+t_{\mathrm{c}}(\mathbf{r})
$$

Again, by numerical subtractions, we can then define spatially resolved kinetic binding energy contributions:

$$
\begin{gathered}
\Delta t_{\mathrm{KS}}(\mathbf{r})=t_{\mathrm{KS}}^{\mathrm{A}}(\mathbf{r})+t_{\mathrm{KS}}^{\mathrm{B}}(\mathbf{r})-t_{\mathrm{KS}}^{\mathrm{AB}}(\mathbf{r}), \\
\Delta t_{\mathrm{tot}}(\mathbf{r})=t_{\mathrm{tot}}^{\mathrm{A}}(\mathbf{r})+t_{\mathrm{tot}}^{\mathrm{B}}(\mathbf{r})-t_{\mathrm{tot}}^{\mathrm{AB}}(\mathbf{r}) .
\end{gathered}
$$

A mapping of the total kinetic energy binding contributions (59) will, in principle, always change if we base the $t_{\text {tot }}$ definition, Eq. (57), on $\tilde{t}_{\mathrm{occ}}$ instead of on $\tilde{t}_{\mathrm{KS}}$, using Eq. (29). This is true even if the integral values $\Delta T_{\mathrm{KS}}$ and $\Delta T_{\text {tot }}$ remain the same. Qualitative differences in the resulting total-kineticenergy mappings are visible for covalent bonding, but not for the cases of noncovalent intermolecular interactions that we have investigated.

The set of top panels of Fig. 3 compares the coupling constant variation in the contributions $\Delta E_{\mathrm{x}, \lambda}$ and $\Delta E_{\mathrm{c}, \lambda}$ to the $\mathrm{H}_{2}, \mathrm{~N}_{2}$, and $\mathrm{O}_{2}$ atomization energies, as computed in vdW-DF-cx. The scaling and the total kinetic-correlation energy contributions vary significantly between these traditional molecular binding examples. The total kinetic-correlation energy contribution to binding $\Delta T_{\mathrm{c}}$ is given by the light blue area under the scaling curve. The value of $\Delta T_{c}$ is dominated by the part that originates from the LDA correlation energy.

The set of bottom panels focus on the coupling-constant scaling of the nonlocal-correlation contribution to the molecular cohesion. The coupling-constant variation in $E_{\mathrm{c}, \lambda}^{\mathrm{nl}}$ can be either upward or downward concave because it is only a part of the kinetic-correlation energy. The upwards and downwards concave behavior corresponds to positive and negative values of $\Delta T_{\mathrm{c}}^{\mathrm{nl}}$ binding contributions, respectively. The dark-blue areas indicate the magnitude of this binding contribution. Reference [106] includes a broad listing and comparisons of molecular-binding contributions $\Delta E_{\mathrm{c}}^{\mathrm{nl}}, \Delta T_{\mathrm{c}}$, and $\Delta T_{\mathrm{c}}^{\mathrm{nl}}[106]$. The comparison also lists $\mathrm{KS}$ binding contributions $\Delta T_{\mathrm{KS}}$, making it clear that the kinetic-correlation energy can only play a significant role in the case of intermolecular binding.

In the case of binding in the $\mathrm{H}_{2}, \mathrm{~N}_{2}$, and $\mathrm{O}_{2}$ molecules, Fig. 3, we observe that the nonlocal-correlation contribution to binding is offset by a contribution to the nonlocal part of the correlation-kinetic energy. As shown in Ref. [106], the same is true for many intramolecular bonds, Tables SI and SII, and for all investigated intermolecular interaction cases, Table SIII.

The binding in the total correlation term, $\Delta E_{\mathrm{c}}$, will be offset by a negative kinetic-correlation energy contribution $\Delta T_{\mathrm{c}}$, as suggested by the virial theorem. However, this need not hold generally for the nonlocal part of the kinetic-correlation energy contribution for intramolecular binding, as further documented in Ref. [106], Tables SI and SII. On the other hand, a compensation can be expected when the nonlocal part of the correlation-kinetic energy is a significant component, such as in most intermolecular interactions. 


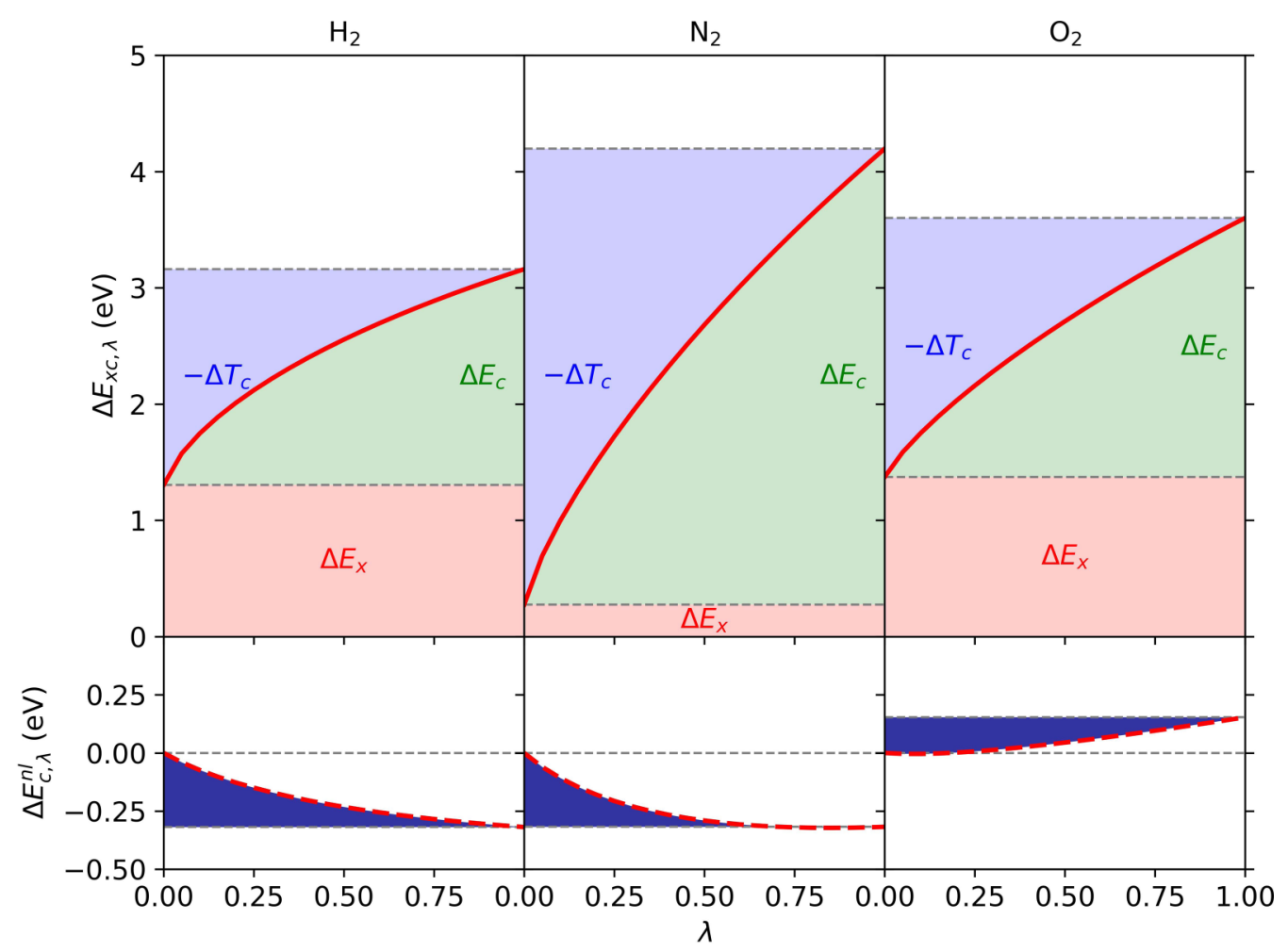

FIG. 3. Coupling-constant scaling for the vdW-DF-cx exchange and correlation contributions to the atomization energy of the $\mathrm{H}_{2}, \mathrm{~N}_{2}$, and $\mathrm{O}_{2}$ molecules. The bottom panel shows the scaling in the nonlocal-correlation contribution to the molecular bindings. The dark blue area is here a measure of the magnitude $\left(108,67\right.$, and $-99 \mathrm{meV}$ for $\mathrm{H}_{2}, \mathrm{~N}_{2}$, and $\left.\mathrm{O}_{2}\right)$ of $\Delta T_{\mathrm{c}}^{\mathrm{nl}}$, i.e., the binding contribution arising in the nonlocal part of the kinetic-correlation energy, Eq. (35).

\section{KINETIC-ENERGY MAPPINGS OF MOLECULAR BINDING}

We analyze and discuss the nature of binding both in $\mathrm{H}_{2}$, $\mathrm{N}_{2}$, and $\mathrm{O}_{2}$ molecules (having traditional chemical bonds) and in noncovalently bonded systems (where, in contrast, there is no pronounced orbital hybridization).

\section{A. Intramolecular interactions}

Figure 4 shows that the kinetic-correlation energy is important in characterizations of intramolecular binding. The figure details the spatial variation in the kinetic-correlation binding energy contribution $\Delta t_{\mathrm{c}}$, in the nonlocal-correlation-kinetic energy contribution $\Delta t_{\mathrm{c}}^{\mathrm{nl}}$, and in the vdW-DF-cx nonlocal correlation energy binding contribution $\Delta e_{\mathrm{c}}^{\mathrm{nl}}$ for the $\mathrm{H}_{2}, \mathrm{~N}_{2}$, and $\mathrm{O}_{2}$ molecules.

We note that the magnitude of the variation in $\Delta t_{\mathrm{c}}$ is about an order-of-magnitude smaller than the KS kinetic-energy binding contribution $\Delta t_{\mathrm{KS}}(\mathbf{r})$ (not shown) for these covalently bonded systems. Nevertheless, there is clear structure in both $\Delta t_{\mathrm{c}}^{\mathrm{nl}}(\mathbf{r})$ and $\Delta e_{\mathrm{c}}^{\mathrm{nl}}(\mathbf{r})$ and a directed nature or signature of vdW interactions even in these strongly bonded dimer molecules.

Reference [106], Tables SI and SII, supported by Fig. S1, provides a broader analysis of such intramolecular bindings. This is done both for the set of molecules for which a PBEbased coupling-constant analysis already exists [11,56], and for the G2-1 benchmark set of molecular atomization energies. Most of these systems are covalently bonded, meaning that orbital hybridization plays the decisive role. However, there are also some G2-1 cases, for example, alkali dimers, where the nonlocal-correlation energy and the nonlocal part of kineticcorrelation energy are important. Here, in the main text, we concentrate on characterizing the binding in $\mathrm{H}_{2}, \mathrm{~N}_{2}$, and $\mathrm{O}_{2}$.

The $\mathrm{O}_{2}$ kinetic-correlation energy binding contribution $\Delta t_{\mathrm{c}}$ deserves a special discussion. The first $\mathrm{O}_{2}$ panel of Fig. 4 shows the variation of $\Delta t_{\mathrm{c}}(\mathbf{r})$ in a plane that contains the binding axis in the dimer. This plot has areas of opposite signs and implies a compensation. However, the overall kinetic-correlation energy contribution is still negative, $\Delta T_{\mathrm{c}}<0$, because the negative regions, away from the axis, have greater weight as we perform the spatial integration. The total, negative kinetic-correlation energy binding contribution is given by the light blue area shown in the left column in Fig. 3.

The second column of Fig. 4 shows the spatial variation in the nonlocal-correlation part of the kinetic-energy binding contribution, $\Delta t_{\mathrm{c}}^{\mathrm{nl}}(\mathbf{r})$, for the three molecules. The variation in $\Delta t_{\mathrm{c}}(\mathbf{r})$ (first column) is generally dominated by the LDA contribution but adjusted by the variation in $\Delta t_{\mathrm{c}}^{\mathrm{nl}}(\mathbf{r})$. The integrated binding contribution from the nonlocal part of the kinetic-correlation energy $\Delta T_{\mathrm{c}}^{\mathrm{nl}}$ can be both negative or positive (as exemplified by the $\mathrm{H}_{2}$ and $\mathrm{N}_{2}$ cases). In such positive- $\Delta T_{\mathrm{c}}^{\mathrm{nl}}$ cases, the binding contribution from $E_{\mathrm{c}}^{\mathrm{nl}}$ is negative, i.e., the nonlocal-correlation energy is actually causing a repulsion in these intramolecular binding cases. Tables SI and SII in Ref. [106] provide a broader overview of the variation in nonlocal-correlation energy and kinetic-correlation energy effects that we document for intramolecular binding. 


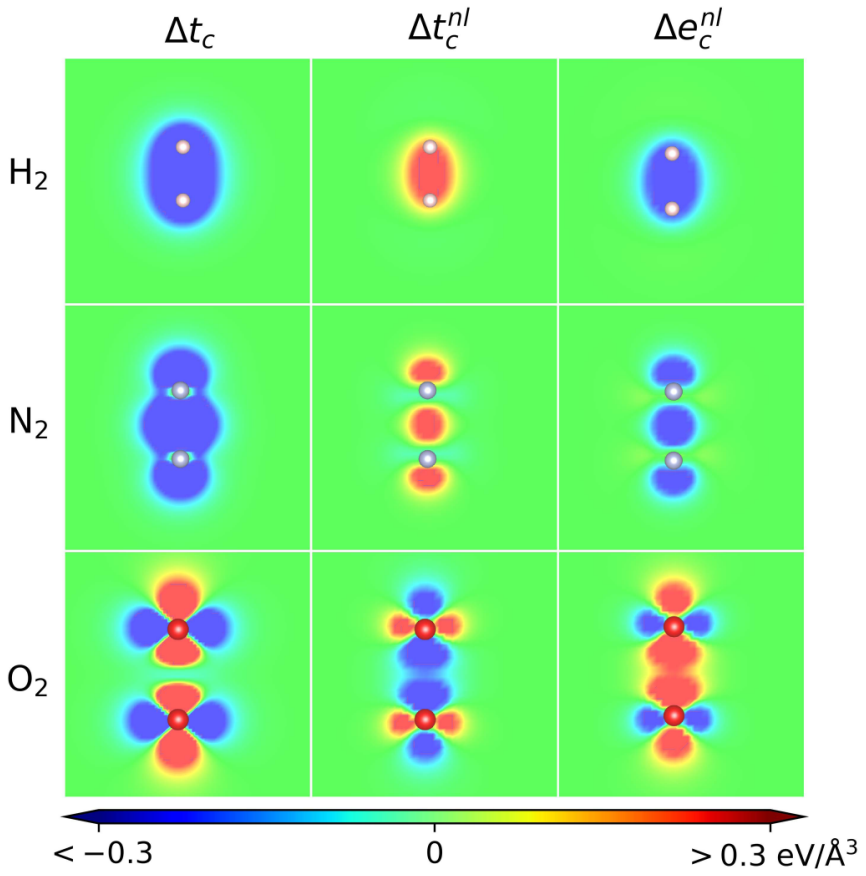

FIG. 4. Spatially resolved kinetic-correlation and nonlocalcorrelation energy contributions to the atomization energy for $\mathrm{H}_{2}$, $\mathrm{N}_{2}$, and $\mathrm{O}_{2}$ molecules. The color map is the energy density in $\mathrm{eV} / \AA^{3}$. The first and second columns show maps of the full kinetic-correlation binding contribution $\Delta t_{\mathrm{c}}(\mathbf{r})$ and of the nonlocal-correlation kinetic energy contribution $\Delta t_{\mathrm{c}}^{\mathrm{nl}}(\mathbf{r})$. The third column shows the bindingenergy contribution $\Delta e_{\mathrm{c}}^{\text {nl }}(\mathbf{r})$ that directly reflects $E_{\mathrm{c}}^{\mathrm{nl}}$.

The third column of Fig. 4 shows a mapping of the nonlocal-correlation energy contribution to binding, $\Delta e_{\mathrm{c}}^{\mathrm{nl}}(\mathbf{r})$, allowing a contrast with the variation documented for $\Delta t_{\mathrm{c}}(\mathbf{r})$ and $\Delta t_{\mathrm{c}}^{\mathrm{nl}}(\mathbf{r})$. We find that $\Delta e_{\mathrm{c}}^{\mathrm{nl}}(\mathbf{r})$ and $\Delta t_{\mathrm{c}}^{\mathrm{nl}}(\mathbf{r})$ are here essentially negative prints of each others. It follows that the full $(\lambda=1)$ nonlocal-correlation energy contribution, given by $\Delta e_{\mathrm{c}}^{\mathrm{nl}}(\mathbf{r})-\Delta t_{\mathrm{c}}^{\mathrm{nl}}(\mathbf{r})$, remains qualitatively described by the variation in $\Delta e_{\mathrm{c}}^{\mathrm{nl}}(\mathbf{r})$ in these cases.

Tables SI and SII in Ref. [106] show that the nonlocalcorrelation energy binding contribution $\Delta E_{\mathrm{c}}^{\mathrm{nl}}$ can take either sign for intramolecular binding. These tables also show that $\Delta T_{\mathrm{c}}^{\mathrm{nl}}$ will typically then have the opposite sign. We therefore generally expect the $\Delta e_{\mathrm{c}}^{\mathrm{nl}}(\mathbf{r})$ and $-\Delta t_{\mathrm{c}}^{\mathrm{nl}}(\mathbf{r})$ contributions to mirror each other, as in Fig. 4. The implication is that $\Delta e_{\mathrm{c}}^{\mathrm{nl}}(\mathbf{r})$ provides a qualitatively correct mapping of the vdW interaction in most covalently bonded cases.

Finally, we note that there are exceptions to this general trend, i.e, cases where $\Delta T_{\mathrm{c}}^{\mathrm{nl}}$ and $\Delta E_{\mathrm{c}}^{\mathrm{nl}}$ are both negative. Figures $S 1$ and $S 2$ in Ref. [106], provide additional analysis for one of these cases, namely $\mathrm{P}_{2}$. In such cases, it is, in principle, necessary to compute both the $\Delta e_{\mathrm{c}}^{\mathrm{nl}}(\mathbf{r})$ and the $\Delta t_{\mathrm{c}}^{\mathrm{nl}}(\mathbf{r})$ variation to obtain a complete mapping, $\Delta e_{\mathrm{c}}^{\mathrm{nl}}(\mathbf{r})-\Delta t_{\mathrm{c}}^{\mathrm{nl}}(\mathbf{r})$, Fig. S2. However, even in these cases, it is still so that $\Delta e_{\mathrm{c}}^{\mathrm{nl}}(\mathbf{r})$ and the $-\Delta t_{\mathrm{c}}^{\mathrm{nl}}(\mathbf{r})$ are approximately mirrors of each others. That is, even in $\mathrm{P}_{2}$, there is no change in the qualitative observations presented above.

\section{B. Intermolecular interactions}

Figure 5 compares kinetic-energy binding contributions $\Delta t_{\mathrm{tot}}(\mathbf{r}), \Delta t_{\mathrm{KS}}(\mathbf{r}), \Delta t_{\mathrm{c}}(\mathbf{r}), \Delta t_{\mathrm{c}}^{\mathrm{nl}}(\mathbf{r})$, in noncovalently bonded systems, i.e., in cases where there is no pronounced orbital hybridization. The top row shows our vdW-DF-cx based results for a benzene dimer, a case which is expected to have an essentially pure vdW (or dispersion) interaction. The bottom row shows results for a water dimer, a case that is predominantly hydrogen bonded, while the middle row explores the mixed-binding benzene-water case.

Table SIII in Ref. [106], supported by Fig. S3, provides a broader characterization of binding in the $\mathrm{S} 22$ benchmark set of such weakly bonded molecular complexes. In all of theses cases, the nonlocal-correlation energy contribution $\Delta E_{\mathrm{c}}^{\mathrm{nl}}$ is positive while $\Delta T_{\mathrm{c}}^{\mathrm{nl}}$ is negative. As such, the following discussion is generic.

For reference, the last column of Fig. 5 shows the spatial variation in the nonlocal-correlation energy binding, $\Delta e_{\mathrm{c}}^{\mathrm{nl}}(\mathbf{r})$. This binding plays a decisive role in all cases. It is a core component of our vdW-DF-cx characterization and it is important for an accurate description of these molecular complexes [18]. In the case of dispersion-bonded systems, the $\Delta e_{\mathrm{c}}^{\mathrm{nl}}(\mathbf{r})$ contributions are the only sources of cohesion; in the case of the water dimer and the benzene-water complexes, there are also significant electrostatic components in the intermolecular interactions.

The first, second, and third columns of Fig. 5 contrast the spatial variation in the binding contributions from the total kinetic energy, from the KS kinetic energy, and from the kinetic-correlation energy. There are no orbital hybridization effects in play, but it is important to note that the $E_{\mathrm{c}}^{\mathrm{nl}}$ binding also pushes densities and thus orbitals around. Smaller signatures are therefore retained in the binding contribution from the KS kinetic energy, $\Delta t_{\mathrm{KS}}(\mathbf{r})$.

Contrasting the panels in the first and second column of Fig. 5, we find that the KS kinetic-energy effects are still the major source of the variation that we compute for $\Delta t_{\mathrm{tot}}(\mathbf{r})$. Nevertheless, in the case of dispersion-bonded systems, we find that there are also important contributions from the kineticcorrelation energy $\Delta t_{\mathrm{c}}(\mathbf{r})$. These contributions, shown in isolation in the third column, arise primarily in the intermolecular region, in areas that have a sparse [33] (but not vanishing) electron density and small-to-moderate density gradient. We sometimes refer to these binding parts as a trough $[6,18]$ but we are then emphasizing the presence of important internal surfaces within such sparse intermolecular regions [14,33,34].

The enhanced binding contributions from internal surfaces reflect the many-electron nature of the vdW problem. The amplitudes of collective (plasmon) excitations are themselves enhanced in the sparse surfacelike intermolecular region and we should then expect larger contributions to the systematic tracking of the electrodynamical coupling among plasmons [1-6,14,15,20]. The vdW enhancement can also be interpreted as reflecting image-plane effects at (internal or external) surfaces [14,38-40,62,108-110], or as multipole response-effects effects when arising outside molecules [25,26,34,35,111-113]. In any case, the vdW-DF-cx handling of screening $[1,3,6,15,24]$ provides mechanisms to track the expected vdW enhancement in the sparse intermolecular regions, at important internal surfaces $[6,14,31,36]$.

The fourth column of Fig. 5 shows the nonlocal part of the kinetic-correlation energy, $\Delta t_{\mathrm{c}}^{\mathrm{nl}}(\mathbf{r})$. Contrasting the third and fourth columns makes it clear that the LDA component of 


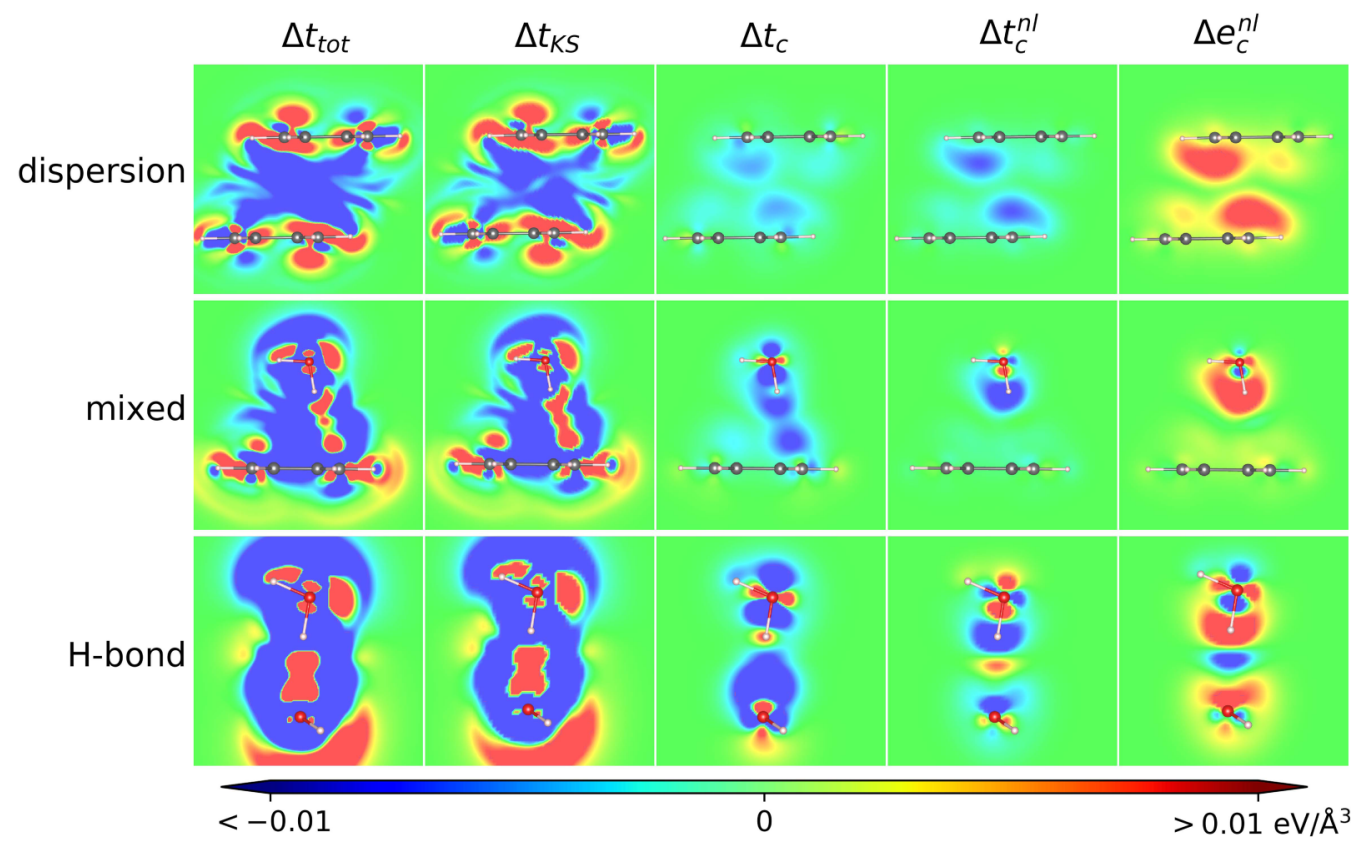

FIG. 5. Spatial variations in binding contributions for the benzene-benzene dimer (first row, identified as "dispersion"), for the water dimer (last row, "H bond"), and for the benzene-water complex (middle row, "mixed"). The panels show contours of the binding-energy density, contrasting the binding contribution arising from the total kinetic energy $\Delta t_{\text {tot }}$ (first column), the dominant KS kinetic-energy component $\Delta t_{\mathrm{KS}}$ (second column), the total kinetic-correlation energy $\Delta t_{\mathrm{c}}$ (third column), and the nonlocal correlation component of the kinetic correlation energy $\Delta t_{\mathrm{c}}^{\mathrm{nl}}$ (fourth column). The latter is found to closely track the spatial variations in the binding contribution from the nonlocal correlation energy variation $\Delta e_{c}^{\mathrm{nl}}$ (last column).

$\Delta t_{\mathrm{c}}(\mathbf{r})$ generally masks the variation in $\Delta t_{\mathrm{c}}^{\mathrm{nl}}(\mathbf{r})$. However, the signatures of the nonlocal kinetic-correlation part dominate in the spare-density regions for dispersion-bonded systems. The nonlocal part also remains a nonvanishing part of full kineticcorrelation energy in the hydrogen-bonded and mixed binding cases.

Figure 6 shows the computed binding contributions in the stacked uracil dimer and in the stacking of adenine and thymine. The top row shows the investigated geometries from the $S 22$ benchmark set [107]. Figure S4 in Ref. [106] shows the variation in the kinetic-energy binding contributions for these systems.

The panels in the bottom two rows of Fig. 6 contrast the variation in the $\Delta e_{\mathrm{c}}^{\mathrm{nl}}$ and $\Delta e_{\mathrm{c}, \lambda=1}^{\mathrm{nl}}=\Delta e_{\mathrm{c}}^{\mathrm{nl}}(\mathbf{r})-\Delta t_{\mathrm{c}}^{\mathrm{nl}}(\mathbf{r})$ accounts of the nonlocal-correlation binding. The contributions are computed for the cuts indicated by the two dashed lines in the top panels. We find that including the nonlocal part of the kinetic-correlation energy enhances the binding signatures found in the $\Delta e_{\mathrm{c}}^{\mathrm{nl}}$ variation in our mapping of the total nonlocal correlation binding, shown in the pair of lower panels. The same is true for the wider set of S22 cases. Comparing the fourth and fifth columns of Fig. 5, we see that they are essentially negative prints of each other, i.e., naturally leading to an enhancement of signatures in $\Delta e_{\mathrm{c}}^{\mathrm{nl}}(\mathbf{r})-\Delta t_{\mathrm{c}}^{\mathrm{nl}}(\mathbf{r})$. For both inter- and intramolecular interactions the variation, $\Delta e_{\mathrm{c}, \lambda=1}^{\mathrm{nl}}(\mathbf{r})$ can effectively be mapped using either $\Delta e_{\mathrm{c}}^{\mathrm{nl}}(\mathbf{r})$ or $-\Delta t_{\mathrm{c}}^{\mathrm{nl}}(\mathbf{r})$.

We also find that the $\Delta e_{\mathrm{c}, \lambda=1}^{\mathrm{nl}}(\mathbf{r})$ signatures are, in effect, channelled into pockets of binding, Figs. 5 and 6 . The dominant contributions are located in the intermolecular (trough) regions but concentrated in areas that resemble orbitals. In other words, we can, in principle, use a similar form of bond-type characterization for a qualitative discussion of the nonlocalcorrelation binding, drawing on an analogy with discussions of chemical bonds.

Before using this vdW-bond mapping analysis, below, we emphasize that this binding is much weaker and that the concentration of binding (in such intermolecular pathways) arises for a different physical reason [16] than in chemical bonds. Chemical binding can exist in the $\lambda \rightarrow 0$ limit (in a Hartree-Fock description) by orbital hybridization, but that cannot happen for the nonlocal-correlation (or vdW) binding. While the inclusion of the $E_{\mathrm{c}}^{\mathrm{nl}}$ energy term in DFT calculations causes density changes, and therefore an electrostatic signature [16], the total nonlocal-correlation binding reflects, instead, an energy gain [16] produced by collective exitations, i.e., by plasmons described by the screening properties $[1,4,6,12,14,15,114]$.

\section{Noble-gas complexes}

Figure 7 contrasts contributions to the dispersion binding in the $\mathrm{Kr}$ dimer (left column of panels) and the $\mathrm{Kr}$ trimer (right column of panels). The results are presented for the optimal structure computed in vdW-DF-cx, Table I, and for the vdWDF-cx solution density.

The top and middle rows of Fig. 7 compare the spatial variations in the total kinetic energy and in the kinetic-correlation energy. The KS kinetic energy effect makes up the larger part in the $\Delta t_{\text {tot }}(\mathbf{r})$ variation because the dispersion interaction leads to density changes and thus to orbitals shifts. However, we find that there are also significant $\Delta t_{\mathrm{c}}(\mathbf{r})$ contributions arising between the noble-gas atoms. 


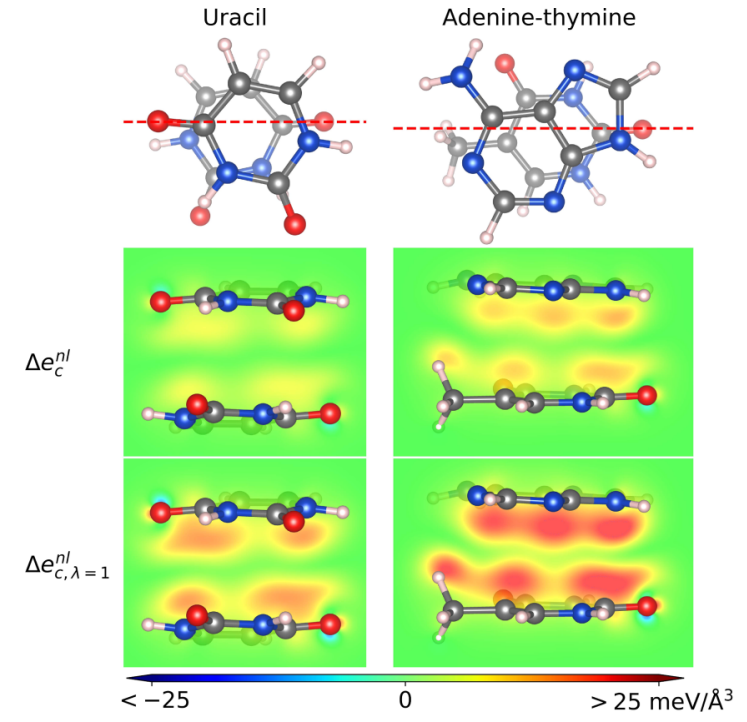

FIG. 6. Spatial variation in nonlocal-correlation binding contributions to the stacked uracil dimer (left) and to the stacking of adenine and thymine bases (right). The panels in the first row show a top view of the configurations; configurations are taken at either the CCSD(T) or MP2 level, from Ref. [107]. The set of red dashed lines marks the planes plotted in which we trace the binding contributions. The panels in the second row show the binding contributions $\Delta e_{\mathrm{c}}^{\mathrm{nl}}$ from the nonlocal correlation energy of the vdW-DF-cx functional. The panels in the third row show the total nonlocal-correlation binding contribution $\Delta e_{\mathrm{c}, \lambda=1}^{\mathrm{nl}}=\Delta e_{\mathrm{c}}^{\mathrm{nl}}-\Delta t_{\mathrm{c}}^{\mathrm{nl}}$, i.e., the spatial variation in the vdW binding of the physical systems, at full electron-electron interaction.

The variation in the $\Delta e_{\mathrm{c}}^{\mathrm{nl}}(\mathbf{r})$ binding contributions, bottom set of panel in Fig. 7, can again be used for a qualitative discussion of the nature of binding. We find (as also documented for the dispersion-bond cases investigated above) that the nonlocal part of the kinetic-correlation energy, $\Delta t_{\mathrm{c}}^{\mathrm{nl}}(\mathbf{r})$ (not shown), reflects the $\Delta t_{\mathrm{c}}(\mathbf{r})$ variation (shown in the middle row of panels) and that $-\Delta t_{\mathrm{c}}^{\mathrm{nl}}(\mathbf{r})$ thus mirrors the variation $\Delta e_{\mathrm{c}}^{\mathrm{nl}}(\mathbf{r})$.

We make two observations about the nature of dispersion binding in such noble gas complexes, based on Fig. 7. First, the vdW-DF method describes the binding as arising in the region between (and not on) the noble-gas atoms. This observation is consistent with a previous vdW-DF characterization of the weak $\mathrm{Ar}_{2}$ charge relocations that arise with the inclusion of the nonlocal correlation term $E_{\mathrm{c}}^{\mathrm{nl}}$, Ref. [16]. However, the vdW-DF-cx picture of dispersion binding is different from the London picture that suggests an atom-centred description $[116,117]$.

Second, the vdW-DF-cx description of binding in the $\mathrm{Kr}$ trimer is not additive, i.e., the vdW-DF-cx account of the $\mathrm{Kr}$ trimer is not merely a sum of dimer contributions. This is evident in Table I and it also holds when freezing the dimers at the slightly longer binding separations that characterize the $\mathrm{Kr}$ trimer.

Our discussion of nonadditivity is based directly on the vdW-DF-cx account of dispersion interactions at binding separation, Fig. 7. Nonadditivity of $\mathrm{vdW}$ forces $[6,34,49,50,111,113,118-126]$ is often discussed in the context of a vdW description that is based on

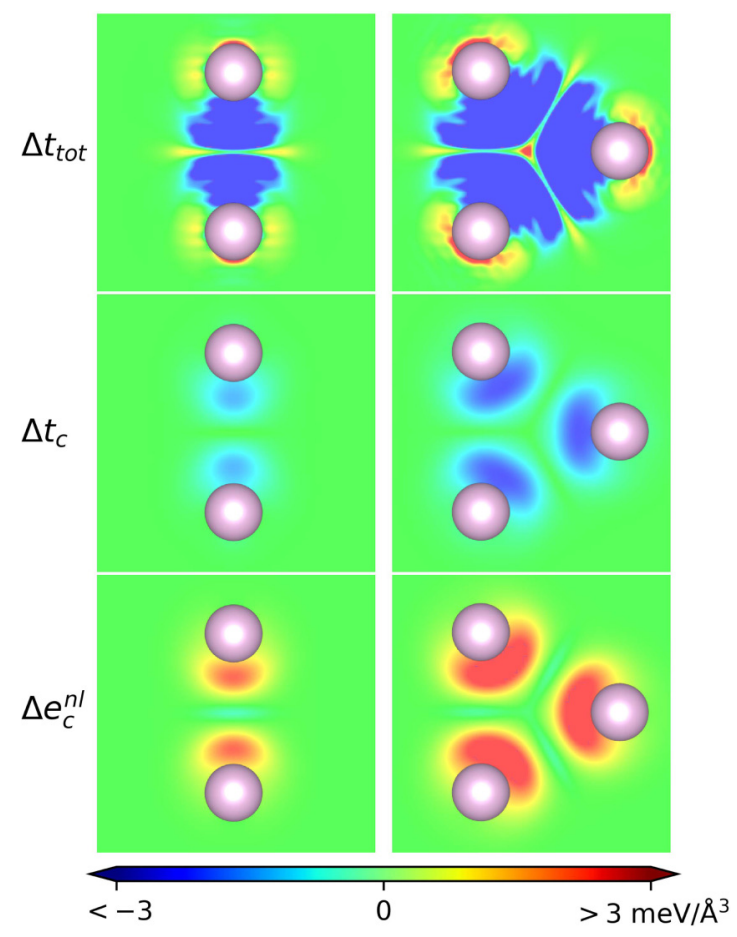

FIG. 7. Spatial variation in contributions to the dispersion binding of the $\mathrm{Kr}$ dimer (left) and of the $\mathrm{Kr}$ trimer (right). The mapping is provided at the vdW-DF-cx results for the optimal structure, Table I. The top, middle, and bottom pair of panels contrast the spatial variation in the total kinetic energy $\Delta\langle\hat{T}\rangle$, the kinetic-correlation energy $\Delta T_{\mathrm{c}}$, and the nonlocal-correlation energy $\Delta E_{\mathrm{c}}^{\mathrm{nl}}$, respectively. The nonlocal part of the kinetic-correlation energy, $\Delta t_{\mathrm{c}}^{\mathrm{nl}}(\mathbf{r}) \approx \Delta t_{\mathrm{c}}(\mathbf{r})$, mirrors the variation in $\Delta e_{\mathrm{c}}^{\mathrm{nl}}(\mathbf{r})$.

adding the series of asymptotic $\mathrm{C}_{6}, \mathrm{C}_{8}, \ldots$ coefficients $[25,26,45,47,112,127-132]$. However, we choose to instead utilize the fact that our $\mathrm{Kr}$ dimer analysis provides us with a spatial mapping of the $\mathrm{vdW}$ bond in a $\mathrm{Kr}$ dimer. This bond is located symmetrically around the axis between the two $\mathrm{Kr}$ atoms. Comparing then such descriptions to that for the $\mathrm{Kr}$ trimer, we note a shift of the vdW binding towards the center region.

Figure 8 provides details of this numerical exploration of the vdW-DF-cx nonadditivity. The figure identifies where the

TABLE I. Bond lengths $d$ and binding energies of noble-gas dimers and trimers, as computed in vdW-DF-cx for fully relaxed structures. Experimental reference values, Ref. [115], are listed in parenthesis when available. The middle column shows our results for the total binding energy, $\Delta E$, of the noble-gas complexes. The last column shows the nonlocal correlation contribution to the binding, $\Delta E_{\mathrm{c}}^{\mathrm{nl}}$.

\begin{tabular}{lccccc}
\hline \hline & \multicolumn{2}{c}{$d(\AA)$} & \multicolumn{2}{c}{$\Delta E(\mathrm{meV})$} & $\Delta E_{\mathrm{c}}^{\mathrm{nl}}(\mathrm{meV})$ \\
\hline Ne dimer & 3.09 & $(3.09)$ & 10.2 & $(3.64)$ & 14.0 \\
Ne trimer & 3.09 & & 30.2 & & 42.8 \\
Ar dimer & 3.99 & $(3.76)$ & 18.9 & $(12.3)$ & 25.7 \\
Ar trimer & 4.02 & & 55.1 & & 75.4 \\
Kr dimer & 4.33 & $(4.01)$ & 22.1 & $(17.3)$ & 30.7 \\
Kr trimer & 4.35 & & 64.6 & & 90.8 \\
\hline \hline
\end{tabular}




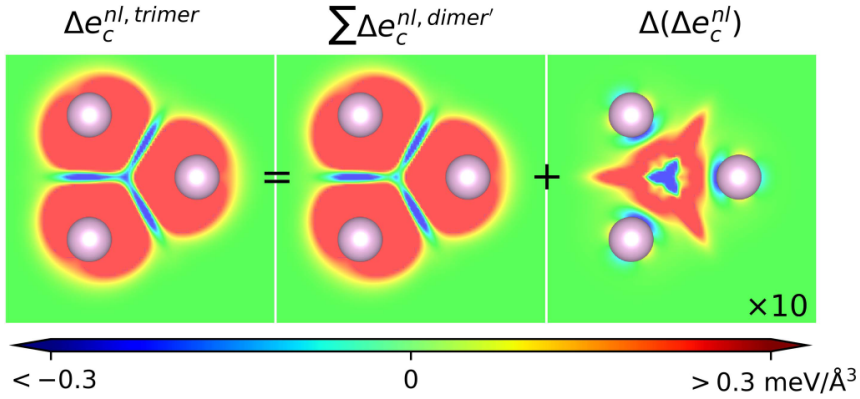

FIG. 8. Nonadditivity of the nonlocal correlation energy binding contributions in the $\mathrm{Kr}$ trimer. The left panel shows the nonlocal correlation contribution to the binding energy in the trimer, directly. The middle panel shows the results of simply making a superposition of the three dimer nonlocal correlation energy binding contributions (evaluated with fixed bond lengths as set by vdW-DF-cx optimization of the $\mathrm{Kr}$ trimer). The right panel shows the spatial variation difference between the actual trimer description and that of the dimer-based superposition, that is, $\Delta\left(\Delta e_{\mathrm{c}}^{\mathrm{nl}}\right)=\Delta e_{\mathrm{c}}^{\mathrm{nl}, \text { trimer }}-\sum \Delta e_{\mathrm{c}}^{\mathrm{nl}, \text { dimer' }}$.

trimer $\Delta e_{\mathrm{c}}^{\mathrm{nl}}$ variation differs from the variation in a sumof-dimer- $\Delta e_{\mathrm{c}}^{\mathrm{nl}}$ description. Interestingly, the trimer binding changes (relative to a sum of dimer contributions), arise in spatially confined pockets in the low-density, small density gradient regions. As such, it further signals the importance of the intermolecular region (of sparse electron distribution) in the description of molecular and other sparse-matter binding.

\section{SUMMARY AND DISCUSSION}

We have provided formal analysis and calculations aiming to deepen the discussion of the nature of $\mathrm{vdW}$ interaction as described in the vdW-DF method. A simple many-body physics effect underpins the vdW interaction, namely the mutual electrodynamical coupling of collective excitations (plamsons) $[1,3,4,6,15]$. This is an effect that exists in the fully interacting (physical) many-body system, described by coupling-constant value $\lambda=1$. The many-body physics effects manifest themselves both in the expectation value of the kinetic energy operator $\hat{T}$ and in the expectation value of the electronelectron interaction operator $\hat{V}$. However, in the standard-and formally exact-KS scheme for DFT calculations, we work with KS kinetic energy while incorporating the remainder, the kinetic-correlation energy $T_{\mathrm{c}}$, within the formulation of an explicit XC functional $E_{\mathrm{xc}}$ (like PBE or vdW-DF-cx).

We observe that a full characterization of the many-body physics effects behind the vdW interactions requires us to identify the $\mathrm{XC}$ contributions at $\lambda=1$ and that such information is available for the vdW-DF by a coupling-constant scaling analysis [8-11,54-56,77]. The $\lambda=1$ system is nominally given by an electron-gas response behavior that corresponds, instead, to the XC functional $E_{\mathrm{xc}}-T_{\mathrm{c}}$. This is one of many consequences of the analysis presented in Refs. [8,10,11,56].

We present a code, called PPACF, so that we can extract spatially resolved binding contributions $\Delta e_{\mathrm{c}, \lambda=1}^{\mathrm{nl}}(\mathbf{r})=$ $\Delta e_{\mathrm{c}}^{\mathrm{nl}}(\mathbf{r})-\Delta t_{\mathrm{c}}^{\mathrm{nl}}(\mathbf{r})$ for this description. We also provide this full-interaction characterization of the nature of the $\mathrm{vdW}$ interaction mechanism $[1,3,4,6]$ for intramolecular binding, for typical intermolecular binding cases of the S22 benchmark set [107], and for a Kr cluster.

Overall, our results for weakly bonded systems confirm that it is the sparse density gradient region between molecules which dominates the contributions to the vdW interactions $[6,14,18,31,34-36,61]$. The vdW interaction is often perceived and handled as an atom-centered effect, i.e., consistent with the original London picture of vdW forces [116,117]. However, as we have also illustrated here, the vdW-DF-cx calculations reveal a different picture.

The vdW-DF ability to handle binding arising in electron tails $[4,6,12,14]$ is important, for it naturally leads to an enhancement of the interaction at binding distances. This is true even if the asymptotic dispersion forces may be weak [20]. In the case of extended systems, this enhancement effect can be interpreted as image-plane effects at external or internal surfaces [14,31,34,36,38-40,62,109,133,134]. For molecules, it is more natural to discuss the binding enhancement through the observation that it is much easier to polarize the electron distribution in the tails than in high-density regions near the atom nuclei $[3,4,6,12,15,25,26,34,35,61]$.

We also highlight that the nonlocal-correlation binding among molecules has signatures, channeled into pockets, i.e., concentrated in regions that resemble an orbital structure. The binding structure, as revealed in $\Delta e_{\mathrm{c}}^{\mathrm{nl}}(\mathbf{r})$ or in $\Delta e_{\mathrm{c}, \lambda=1}^{\mathrm{nl}}(\mathbf{r})$, shares characteristics that resemble (but is much weaker than) those found in the KS kinetic-energy account of traditional chemical binding. This observation is useful for developing the qualitative discussions of vdW forces. For example, we can use such bond signatures to document that the vdW-DF-cx account of the nonlocal-correlation binding in the $\mathrm{Kr}$ trimer is not additive - the binding is not merely a sum nonlocal-correlation binding contributions in $\mathrm{Kr}$ dimers.

Finally, we note that our coupling constant scaling results allow us to generalize the construction of strictly parameterfree ACF-based hybrids [9-11,55,56] to a foundation in the vdW-DF method. Such constructions and the implications for the use of the vdW-DF-cx0 hybrid [70] is presented in a forthcoming paper.

\section{ACKNOWLEDGMENTS}

We thank Kristian Berland and Jeffrey B. Neaton for useful discussions. Work supported by the Swedish Research Council (VR) through Grants No. 2014-4310 and No. 2014-5289 and the Chalmers Area-of-Advance-Materials theory activity. The authors also acknowledge computer allocations from the Swedish National Infrastructure for Computing (SNIC), under Contract SNIC2016-10-12 and SNIC 2017/1-174, and from the Chalmers Centre for Computing, Science and Engineering (C3SE) under Contract C3SE2017-1-3.

\section{APPENDIX: COUPLING CONSTANT SCALING}

The coupling-constant result (22) can be obtained from a renormalization-group perspective on the ACF. In the coupling-constant analysis of $\mathrm{XC}$ functionals [8-11,55,56,76,77], we consider a would-be many-body physics problem specified by the Hamiltonian

$$
\hat{H}_{\alpha}=\hat{T}+\alpha \hat{V}+V_{\text {ext }},
$$


where $\hat{V}$, again, denotes the operator for full (or actual) electron-electron interaction, and where $V_{\text {ext }}$ is the external potential, in part set by the nuclei. The formal machinery of DFT [7,135] works for $\hat{H}_{\alpha}$ as well as it does for the actual, physical problem defined by $\hat{H}=\hat{H}_{\alpha=1}$. In such a generalized-DFT framework, we use $\Psi_{n}^{\min , \alpha}$ to denote the ground-state many-body wave function solution, which at any given $\alpha$ will be a unique functional of the density variation $n(\mathbf{r})$, as indicated. Also, $\Psi_{n}^{\mathrm{min}}$ denotes the ground-state solution for the physical problem, at $\alpha=1$, i.e., the problem for which we normally employ the DFT construction using a KS calculational scheme [7].

For any given $\gamma$, we can consider the density scaling $n(\mathbf{r}) \rightarrow$ $\gamma^{3} n(\gamma \mathbf{r})$, where the choice $\gamma=1 / \lambda$ corresponds to the scaling that was discussed in the main text. The key observation [8] is that this scaling permits us to formally construct the couplingconstant scaling in the ground-state many-body wave-function solutions to $\hat{H}_{\alpha}$,

$$
\Psi_{n}^{\min , \alpha}\left(\mathbf{r}_{1}, \ldots, \mathbf{r}_{N}\right)=\alpha^{3 N / 2} \Psi_{n_{\gamma}}^{\min }\left(\alpha \mathbf{r}_{1}, \ldots, \alpha \mathbf{r}_{N}\right),
$$

for $\gamma=\alpha^{-1}$, Ref. [9]. We note that $\Psi_{n_{\gamma}}^{\min }$ is a ground-state solution for the density $n_{\gamma=1 / \alpha}$. The KS kinetic-energy functional is defined as [9]

$$
T_{\mathrm{KS}}[n]=\left\langle\Phi_{n}^{\min }|\hat{T}| \Phi_{n}^{\min }\right\rangle,
$$

where $\Phi_{n}^{\min }$ is a Slater-determinant wave function that corresponds to the density variation $n(\mathbf{r})$ and minimizes the expectation value in Eq. (A3).

For any assumed value of $\alpha$, a generalization of the XC standard energy functional,

$$
E_{\mathrm{xc}}^{\alpha}[n] \equiv\left\langle\Psi_{n}^{\min , \alpha}|(\hat{T}+\alpha \hat{V})| \Psi_{n}^{\min , \alpha}\right\rangle-T_{\mathrm{KS}}[n]-\alpha U[n],
$$

will permit us to pursue DFT calculations in a generalized KS scheme, solving the $\hat{H}_{\alpha}$ problem $[2,7,11,76]$. In Eq. (A4), $U[n]$ denotes the mean-field Coulomb interaction among electrons, Eq. (30). Similar as for the wave functions, we reserve the subscript-free version $E_{\mathrm{xc}}[n]$ to denote the standard $\mathrm{XC}$ energy functional, i.e., relevant for the standard KS scheme in DFT [7].

Following Refs. [8,11,76], we further define $\alpha$-specific density functionals for the expectation values of the electronelectron interaction,

$$
V_{e-e}^{\alpha}[n] \equiv\left\langle\Psi_{n}^{\min , \alpha}|\hat{V}| \Psi_{n}^{\min , \alpha}\right\rangle,
$$

and the expectation value of the kinetic-energy operator,

$$
T^{\alpha}[n] \equiv\left\langle\Psi_{n}^{\min , \alpha}|\hat{T}| \Psi_{n}^{\min , \alpha}\right\rangle .
$$

At any given density $n$, it follows that $\alpha U[n]=\alpha^{2} U\left[n_{1 / \alpha}\right]$, $T_{\mathrm{KS}}[n]=\alpha^{2} T_{\mathrm{KS}}\left[n_{1 / \alpha}\right]$ and that

$$
T^{\alpha}[n]+\alpha V_{e-e}^{\alpha}[n]=\alpha^{2}\left\langle\Psi_{n_{\gamma}}^{\min }|(\hat{T}+\hat{V})| \Psi_{n_{\gamma}}^{\min }\right\rangle,
$$

for $\gamma=1 / \alpha$.

Next, we revisit the ACF [2,28,29], Eq. (9), noting that we might just as well use it to define and compute the $\alpha$-specific $\mathrm{XC}$ functional

$$
E_{\mathrm{xc}}^{\alpha}[n]=\int_{0}^{\alpha} d \lambda E_{\mathrm{xc}, \lambda}[n] .
$$

This XC functional can be used in a KS scheme for solving the $\hat{H}_{\alpha}$ ground-state problem $[2,7,8,76]$. The density-scaling result (A7) implies that such $\mathrm{XC}$ energy functional adheres to a simple scaling result $[8,76]$,

$$
E_{\mathrm{xc}}^{\alpha}[n]=\alpha^{2} E_{\mathrm{xc}}\left[n_{1 / \alpha}\right] .
$$

Finally, the main scaling result for the XC energy functional, Eq. (22), follows from the renormalization condition, Eq. (A8), by simple derivation in the assumed value of the coupling constant in $\hat{H}_{\alpha}$.
[1] G. D. Mahan, J. Chem. Phys. 43, 1569 (1965).

[2] D. C. Langreth and J. P. Perdew, Phys. Rev. B 15, 2884 (1977).

[3] K. Rapcewicz and N. W. Ashcroft, Phys. Rev. B 44, 4032 (1991).

[4] A. C. Maggs and N. W. Ashcroft, Phys. Rev. Lett. 59, 113 (1987).

[5] D. C. Langreth and S. H. Vosko, Phys. Rev. Lett. 59, 497 (1987).

[6] P. Hyldgaard, K. Berland, and E. Schröder, Phys. Rev. B 90, 075148 (2014).

[7] W. Kohn and L. J. Sham, Phys. Rev. 140, A1133 (1965).

[8] M. Levy and J. P. Perdew, Phys. Rev. A 32, 2010 (1985).

[9] M. Levy, Phys. Rev. A 43, 4637 (1991).

[10] A. Görling and M. Levy, Phys. Rev. B 47, 13105 (1993).

[11] K. Burke, M. Ernzerhof, and J. P. Perdew, Chem. Phys. Lett. 265, 115 (1997).

[12] Y. Andersson, D. C. Langreth, and B. I. Lundqvist, Phys. Rev. Lett. 76, 102 (1996).

[13] H. Rydberg, B. I. Lundqvist, D. C. Langreth, and M. Dion, Phys. Rev. B 62, 6997 (2000).
[14] H. Rydberg, M. Dion, N. Jacobson, E. Schröder, P. Hyldgaard, S. I. Simak, D. C. Langreth, and B. I. Lundqvist, Phys. Rev. Lett. 91, 126402 (2003).

[15] M. Dion, H. Rydberg, E. Schröder, D. C. Langreth, and B. I. Lundqvist, Phys. Rev. Lett. 92, 246401 (2004).

[16] T. Thonhauser, V. R. Cooper, S. Li, A. Puzder, P. Hyldgaard, and D. C. Langreth, Phys. Rev. B 76, 125112 (2007).

[17] K. Lee, È. D. Murray, L. Kong, B. I. Lundqvist, and D. C. Langreth, Phys. Rev. B 82, 081101 (2010).

[18] K. Berland and P. Hyldgaard, Phys. Rev. B 89, 035412 (2014).

[19] T. Thonhauser, S. Zuluaga, C. A. Arter, K. Berland, E. Schröder, and P. Hyldgaard, Phys. Rev. Lett. 115, 136402 (2015).

[20] K. Berland, V. R. Cooper, K. Lee, E. Schröder, T. Thonhauser, P. Hyldgaard, and B. I. Lundqvist, Rep. Prog. Phys. 78, 066501 (2015).

[21] J. P. Perdew, K. Burke, and M. Ernzerhof, Phys. Rev. Lett. 77, 3865 (1996). 
[22] J. P. Perdew, A. Ruzsinszky, G. I. Csonka, O. A. Vydrov, G. E. Scuseria, L. A. Constantin, X. Zhou, and K. Burke, Phys. Rev. Lett. 100, 136406 (2008).

[23] H. Rydberg, Nonlocal correlations in density functional theory, Ph.D. thesis, Department of Applied Physics, Chalmers University of Technology, Göteborg, Sweden, 2001, http://bitmath.se/rydberg/Thesis.

[24] K. Berland, C. A. Arter, V. R. Cooper, K. Lee, B. I. Lundqvist, E. Schröder, T. Thonhauser, and P. Hyldgaard, J. Chem. Phys. 140, 18A539 (2014).

[25] A. D. Becke and E. R. Johnson, J. Chem. Phys. 123, 154101 (2005).

[26] A. D. Becke and E. R. Johnson, J. Chem. Phys. 127, 154108 (2007).

[27] G. Román-Pérez and J. M. Soler, Phys. Rev. Lett. 103, 096102 (2009).

[28] D. C. Langreth and J. P. Perdew, Solid State Commun. 17, 1425 (1975).

[29] O. Gunnarsson and B. I. Lundqvist, Phys. Rev. B 13, 4274 (1976).

[30] M. Callsen, N. Atodiresei, V. Caciuc, and S. Blügel, Phys. Rev. B 86, 085439 (2012).

[31] P. Lazić, N. Atodiresei, V. Caciuc, R. Brako, B. Gumhalter, and S. Blügel, J. Phys.: Condens. Matter 24, 424215 (2012).

[32] M. Dion, H. Rydberg, E. Schröder, D. C. Langreth, and B. I. Lundqvist, Phys. Rev. Lett. 95, 109902(E) (2005).

[33] D. C. Langreth, B. I. Lundqvist, S. D. Chakarova-Käck, V. R. Cooper, M. Dion, P. Hyldgaard, A. Kelkkanen, J. Kleis, L. Kong, S. Li, P. G. Moses, E. Murray, A. Puzder, H. Rydberg, E. Schröder, and T. Thonhauser, J. Phys.: Condens. Matter 21, 084203 (2009).

[34] J. Kleis, E. Schröder, and P. Hyldgaard, Phys. Rev. B 77, 205422 (2008).

[35] K. Berland and P. Hyldgaard, J. Chem. Phys. 132, 134705 (2010).

[36] K. Berland and P. Hyldgaard, Phys. Rev. B 87, 205421 (2013).

[37] E. M. Lifshitz, Zh. Eksp. Teor. Fiz. 29, 94 (1956) [Sov. Phys. JETP 2, 73 (1956)].

[38] E. Zaremba and W. Kohn, Phys. Rev. B 13, 2270 (1976).

[39] E. Zaremba and W. Kohn, Phys. Rev. B 15, 1769 (1977).

[40] P. Nordlander and J. Harris, J. Phys. C 17, 1141 (1984).

[41] E. Hult, P. Hyldgaard, J. Rossmeisl, and B. I. Lundqvist, Phys. Rev. B 64, 195414 (2001).

[42] J. Kleis, B. I. Lundqvist, D. C. Langreth, and E. Schröder, Phys. Rev. B 76, 100201 (2007).

[43] S. Grimme, J. Comput. Chem. 25, 1463 (2004).

[44] S. Grimme, J. Chem. Phys. 124, 034108 (2006).

[45] S. Grimme, J. Antony, S. Ehrlich, and H. Krieg, J. Chem. Phys. 132, 154104 (2010).

[46] P. L. Silvestrelli, Phys. Rev. Lett. 100, 053002 (2008).

[47] A. Tkatchenko and M. Scheffler, Phys. Rev. Lett. 102, 073005 (2009).

[48] O. A. Vydrov and T. Van Voorhis, J. Chem. Phys. 133, 244103 (2010).

[49] V. G. Ruiz, W. Liu, E. Zojer, M. Scheffler, and A. Tkatchenko, Phys. Rev. Lett. 108, 146103 (2012).

[50] A. Tkatchenko, R. A. DiStasio, R. Car, and M. Scheffler, Phys. Rev. Lett. 108, 236402 (2012).

[51] J. P. Perdew, K. Burke, and Y. Wang, Phys. Rev. B 54, 16533 (1996).
[52] B. I. Lundqvist, Y. Andersson, H. Shao, S. Chan, and D. C. Langreth, Int. J. Quantum Chem. 56, 247 (1995).

[53] J. F. Dobson and B. P. Dinte, Phys. Rev. Lett. 76, 1780 (1996).

[54] M. Levy, in Density Functional Theory, edited by E. K. U. Gross and R. M. Dreizler (Plenum Press, New York, 1995), pp. 11-31.

[55] J. P. Perdew, M. Ernzerhof, and K. Burke, J. Chem. Phys. 105, 9982 (1996).

[56] M. Ernzerhof, J. P. Perdew, and K. Burke, Int. J. Quantum Chem. 64, 285 (1997).

[57] V. R. Cooper, Phys. Rev. B 81, 161104 (2010).

[58] J. Klimeš, D. R. Bowler, and A. Michaelides, J. Phys.: Condens. Matter 22, 022201 (2010).

[59] J. Klimeš, D. R. Bowler, and A. Michaelides, Phys. Rev. B 83, 195131 (2011).

[60] I. Hamada, Phys. Rev. B 89, 121103 (2014).

[61] K. Berland, Ø. Borck, and P. Hyldgaard, Comput. Phys. Commun. 182, 1800 (2011).

[62] K. Lee, A. K. Kelkkanen, K. Berland, S. Andersson, D. C. Langreth, E. Schröder, B. I. Lundqvist, and P. Hyldgaard, Phys. Rev. B 84, 193408 (2011).

[63] K. Lee, B. Kolb, T. Thonhauser, D. Vanderbilt, and D. C. Langreth, Phys. Rev. B 86, 104102 (2012).

[64] D. C. Langreth and J. P. Perdew, Phys. Rev. B 21, 5469 (1980).

[65] D. C. Langreth and M. J. Mehl, Phys. Rev. Lett. 47, 446 (1981).

[66] D. C. Langreth and S. H. Vosko, Adv. Quantum Chem. 21, 175 (1990).

[67] L. Hedin and B. I. Lundqvist, J. Phys. C 4, 2064 (1971).

[68] J. P. Perdew and Y. Wang, Phys. Rev. B 46, 12947 (1992).

[69] C. Adamo and V. Barone, J. Chem. Phys. 110, 6158 (1999).

[70] K. Berland, Y. Jiao, J.-H. Lee, T. Rangel, J. B. Neaton, and P. Hyldgaard, J. Chem. Phys. 146, 234106 (2017).

[71] P. Giannozzi, S. Baroni, N. Bonini, M. Calandra, R. Car, C. Cavazzoni, D. Ceresoli, G. L. Chiarotti, M. Cococcioni, I. Dabo, A. D. Corso, S. de Gironcoli, S. Fabris, G. Fratesi, R. Gebauer, U. Gerstmann, C. Gougoussis, A. Kokalj, M. Lazzeri, L. Martin-Samos, N. Marzari, F. Mauri, R. Mazzarello, S. Paolini, A. Pasquarello, L. Paulatto, C. Sbraccia, S. Scandolo, G. Sclauzero, A. P. Seitsonen, A. Smogunov, P. Umari, and R. M. Wentzcovitch, J. Phys.: Condens. Matter 21, 395502 (2009).

[72] M. von Hopffgarten and G. Frenking, WIREs Comput. Mol. Sci. 2, 43 (2012).

[73] M. W. Schmidt, J. Ivanic, and K. Ruedenberg, J. Chem. Phys. 140, 204104 (2014).

[74] J. Rohrer and P. Hyldgaard, Phys. Rev. B 83, 165423 (2011).

[75] M. Rahm and R. Hoffmann, J. Am. Chem. Soc. 138, 3731 (2016).

[76] M. Levy, W. Yang, and R. G. Parr, J. Chem. Phys. 83, 2334 (1985).

[77] M. Levy, N. H. March, and N. C. Handy, J. Chem. Phys. 104, 1989 (1996).

[78] T. Rangel, K. Berland, S. Sharifzadeh, F. Brown-Altvater, K. Lee, P. Hyldgaard, L. Kronik, and J. B. Neaton, Phys. Rev. B 93, 115206 (2016).

[79] F. Brown-Altvater, T. Rangel, and J. B. Neaton, Phys. Rev. B 93, 195206 (2016).

[80] A. Ambrosetti and P. L. Silvestrelli, Phys. Rev. B 94, 045124 (2016). 
[81] L. Gharaee, P. Erhart, and P. Hyldgaard, Phys. Rev. B 95, 085147 (2017).

[82] R. Petuya and A. Arnau, Carbon 116, 599 (2017).

[83] I. Loncaric, J. Popovic, V. Despoja, S. Burazer, I. Grgicevic, D. Popovic, and Z. Skoko, Cryst. Growth Des. 17, 4445 (2017).

[84] P. A. T. Olsson, E. Schröder, P. Hyldgaard, M. Kroon, E. Andreasson, and E. Bergvall, Polymer 121, 234 (2017).

[85] M. Wang, J.-Q. Zhong, J. Kestell, I. Waluyo, D. J. Stacchiola, A. J. Boscoboinik, and D. Lu, Top. Catal. 60, 481 (2017).

[86] X. Wang, K. Esfarjani, and M. Zebarjadi, J. Phys. Chem. C 121, 15529 (2017).

[87] T. Björkman, J. Chem. Phys. 141, 074708 (2014).

[88] P. Erhart, P. Hyldgaard, and D. Lindroth, Chem. Mater 27, 5511 (2015).

[89] H. Sadeghi, S. Santarash, and C. J. Lambert, Sci. Rep. 5, 9514 (2015).

[90] R. C. Clay, M. Holzmann, D. M. Ceperley, and M. A. Morales, Phys. Rev. B 93, 035121 (2016).

[91] J. Löfgren, H. Grönbeck, L. Moth-Poulsen, and P. Erhart, Phys. Rev. B 94, 115205 (2016).

[92] M. Fritz, M. Fernandez-Serra, and J. M. Soler, J. Chem. Phys. 144, 224101 (2016).

[93] S. Javaid and M. J. Akhtar, Chem. Phys. Lett. 649, 73 (2016).

[94] B. Borca, V. Schendel, R. Petuya, I. Pentegov, T. Michnowicz, U. Kraft, H. Klauk, A. Arnau, P. Wahl, U. Schlickum, and K. Kern, ACS Nano 9, 12506 (2015).

[95] M. Hellström, I. Beinik, P. Broqvist, J. V. Lauritsen, and K. Hermansson, Phys. Rev. B 94, 245433 (2016).

[96] M. Mehboudi, A. M. Dorio, W. Zhu, A. van der Zande, H. O. H. Churchill, A. A. Pacheco-Sanjuan, E. O. Harriss, P. Kumar, and S. Baraza-Lopez, Nano Lett. 16, 1704 (2016).

[97] M. Mehboudi, B. M. Fregoso, Y. Yang, W. Zhu, A. van der Zande, J. Ferrer, L. Bellaiche, P. Kumar, and S. Barraza-Lopez, Phys. Rev. Lett. 117, 246802 (2016).

[98] J. Löfgren, H. Grönbeck, L. Moth-Poulsen, and P. Erhart, J. Phys. Chem. C 120, 12059 (2016).

[99] E. Kuisma, C. F. Hansson, T. B. Lindberg, C. A. Gillberg, S. Idh, and E. Schröder, J. Chem. Phys. 144, 184704 (2016).

[100] J. Čechal, C. S. Kley, R. Pétuya, F. Schramm, M. Ruben, S. Stepanow, A. Sebastian, A. Arnau, and K. Kern, J. Phys. Chem. C 120, 18622 (2016).

[101] J.-Q. Zhong, M. Wang, N. Akter, J. D. Kestell, A. M. Boscoboinik, T. Kim, D. J. Stacchiola, D. Lu, and A. J. Boscoboinik, Nat. Commun. 8, 16118 (2017).

[102] B. Borca, T. Michnowicz, R. Petuya, M. Pristl, V. Schendel, I. Pentegov, U. Kraft, H. Klauk, P. Wahl, R. Gutzler, A. Arnau, U. Schlickum, and K. Kern, ACS Nano 11, 4703 (2017).

[103] I. Brihuega and F. Yndurain, J. Phys. Chem. B 122, 595 (2018).

[104] G. G. Kebede, D. Spångberg, P. D. Mitev, P. Broqvist, and K. Hermansson, J. Chem. Phys. 146, 064703 (2017).

[105] Ø. Borck and E. Schröder, Surf. Sci. 664, 162 (2017).

[106] See Supplemental Material at http://link.aps.org/supplemental/ 10.1103/PhysRevB.97.085115 for a broad characterization and additional analysis of intramolecular and intermolecular binding.

[107] P. Jurecka, J. Sponer, J. Cerny, and P. Hobza, Phys. Chem. Chem. Phys. 8, 1985 (2006).
[108] See, for example, M. Persson and S. Andersson, in Handbook of Surface Science, edited by E. Hasselbrink and B. I. Lundqvist (Elsevier, Amsterdam, 2008), Vol. 3, Chap. 4, p. 95.

[109] K. Lee, K. Berland, M. Yoon, S. Andersson, E. Schröder, P. Hyldgaard, and B. I. Lundqvist, J. Phys.: Condens. Matter 24, 424213 (2012).

[110] J. Tao and A. M. Rappe, Phys. Rev. Lett. 112, 106101 (2014).

[111] J. Tao and J. P. Perdew, J. Chem. Phys. 141, 141101 (2014).

[112] J. Tao, J. Yang, and A. M. Rappe, J. Chem. Phys. 142, 164302 (2015).

[113] J. Tao, Y. Jiao, Y. Mo, Z.-H. Yang, J.-X. Zhu, P. Hyldgaard, and J. P. Perdew [Phys. Rev. B (to be published)], arXiv:1711.00655.

[114] D. C. Langreth, M. Dion, H. Rydberg, E. Schröder, P. Hyldgaard, and B. I. Lundqvist, Int. J. Quantum Chem. 101, 599 (2005).

[115] J. Ogilvie and F. Y. Wang, J. Mol. Struct. 273, 277 (1992).

[116] F. London, Z. Phys. 63, 245 (1930).

[117] F. London, Z. Physik. Chemie 33, 8 (1937), english translations in H. Hettema, Quantum Chemistry, Classic Scientific Papers (World Scientific, Singapore, 2000); F. London, Trans. Faraday Soc. 33, 8b (1937).

[118] Y. S. Barash and V. L. Ginzburg, Usp. Fiz. Nauk. 143, 345 (1984) [Sov. Phys. Usp. 27, 467 (1984)].

[119] Y. S. Barash, Fiz. Tverd. Tela (Leningrad) 30, 2738 (1988) [Sov. Phys. Solid State 30, 1578 (1988)].

[120] B. E. Sernelius and P. Björk, Phys. Rev. B 57, 6592 (1998).

[121] J. F. Dobson, A. White, and A. Rubio, Phys. Rev. Lett. 96, 073201 (2006).

[122] S. Lebègue, J. Harl, T. Gould, J. G. Ángyán, G. Kresse, and J. F. Dobson, Phys. Rev. Lett. 105, 196401 (2010).

[123] J. Klimeš and A. Michaelides, J. Chem. Phys. 137, 120901 (2012).

[124] J. F. Dobson and T. Gould, J. Phys.: Condens. Matter 24, 073201 (2012).

[125] J. F. Dobson, T. Gould, and G. Vignale, Phys. Rev. X 4, 021040 (2014).

[126] J. F. Dobson, Intl. J. Quantum Chem. 114, 1157 (2014).

[127] S. Grimme, WIREs: Comput. Mol. Sci. 1, 211 (2011).

[128] A. Tkatchenko, L. Romaner, O. T. Hofmann, E. Zojer, C. Ambrosch-Draxl, and M. Scheffler, MRS Bull. 35, 435 (2010).

[129] J. P. Perdew, A. Ruzsinszky, J. Sun, S. Glindmeyer, and G. I. Csonka, Phys. Rev. A 86, 062714 (2012).

[130] J. P. Perdew, J. Tao, P. Hao, A. Ruzsinszky, G. I. Csonka, and J. M. Pitarke, J. Phys.: Condens. Matter 24, 424207 (2012).

[131] A. Ruzsinszky, J. P. Perdew, J. Tao, G. I. Csonka, and J. M. Pitarke, Phys. Rev. Lett. 109, 233203 (2012).

[132] J. Tao and A. M. Rappe, J. Chem. Phys. 144, 031102 (2016).

[133] K. Berland, T. L. Einstein, and P. Hyldgaard, Phys. Rev. B 80, 155431 (2009).

[134] K. Berland, Connected by voids: Interactions and screening in sparse matter, Ph.D. thesis, Department of Microtechnology and Nanoscience - MC2, Chalmers University of Technology, Göteborg, Sweden, 2012.

[135] P. Hohenberg and W. Kohn, Phys. Rev. 136, B864 (1964). 